Real Analysis Exchange

Vol. 23(2), 1997-1998, pp. 491-524

Vasile Ene, Ovidius University Constanța, Romania

Current address: 23 August 8717, Jud. Constanţa, Romania

e-mail: ene@s23aug.sfos.ro or ene@univ-ovidius.ro

\title{
HAKE-ALEXANDROFF-LOOMAN TYPE THEOREMS
}

\begin{abstract}
In this paper we shall study three kinds of descriptive type integrals, that all generalize the wide Denjoy integral. In fact the classes of primitives for these integrals, restricted to the continuous functions, are $A C G$. We shall also study five kinds of Perron type integrals, that are all in a close relationship with the descriptive type integrals. In the last three sections we show some relationships between the descriptive type integrals and the Perron type integrals.
\end{abstract}

\section{Introduction}

To define descriptive type integrals on compact intervals the following two facts are essential.

1) To have a sufficiently general monotonicity theorem.

2) To find some linear spaces, sufficiently general, such that the monotonicity theorem can be applied.

In this paper we shall study three kinds of descriptive type integrals that all generalize the wide Denjoy integral. In fact the classes of primitives for these integrals, restricted to the continuous functions, are $A C G$.

The integrals mentioned above are based on the following facts.

(I) $[A C G]$ on a compact set is a linear space.

(II) $[V B G] \cap(N)$ on a compact set is a linear space ([23], [3], [4]).

(III) $V B G \cap(N)$ for Borel functions on a Borel set is a linear space ([3], [4]).

Key Words: $A C G, V B G$, Lusin's condition $(N)$, Foran's condition $(M)$, nonabsolutely convergent integrals

Mathematical Reviews subject classification: 26A39, 26A45, 26A46

Received by the editors June 30, 1997

*I wish to thank the referee for careful reading and valuable suggestions. 
In [11], C. M. Lee introduced the very abstract $\mathcal{L} D G$ integral, using (I) and his monotonicity Theorem A, a). The integrals based on (II) and (III) use Theorem A, b).

To define Perron type integrals on compact intervals the following two facts are essential.

$\left.1^{\prime}\right)$ To have a sufficiently general monotonicity theorem.

$\left.2^{\prime}\right)$ To find some upper semilinear spaces, sufficiently general, such that the monotonicity theorem can be applied.

In this paper we shall study five kinds of Perron type integrals, that are all in a close relationship with the descriptive type integrals. These integrals are based on the following facts.

$(\mathrm{I})^{\prime}[\underline{A C G}],[\underline{L} G]$ and $[(A C \cap \underline{L}) G]$ on a compact set are upper semilinear spaces.

$(\mathrm{II})^{\prime}[V B G] \cap(\underline{N})$ on a compact set is an upper semilinear space $([4])$.

$(\mathrm{III})^{\prime} \quad V B G \cap(\underline{N})$ for Borel functions on a Borel set is an upper semilinear space $([4])$.

In [11], C. M. Lee introduced the very abstract $\mathcal{L} P G$ integral, using $[\underline{A C G}]$ and Theorem A, a). The Perron type integrals based on (II) ${ }^{\prime}$ and (III) ${ }^{\prime}$ use Theorem A, c).

The Hake-Alexandroff-Looman Theorem asserts that the restricted Denjoy integral is equivalent to the classical Perron integral (see [19], pp. 247-252). In what follows, by a Hake-Alexandroff-Looman type theorem we mean a theorem that establishes the equivalence between a descriptive type integral and a Perron type integral.

In the last three sections we show some relationships between the descriptive type integrals and the Perron type integrals. We obtain in Corollary 6 that C. M. Lee's $\mathcal{L} P G$ integral is a strict generalization of his $\mathcal{L} D G$ integral (although he claimed in [11] that they were equivalent). In general the descriptive integrals (II) are strictly contained in the Perron type integrals (II)' but we identify situations in which the two integrals are equivalent. We show that the descriptive integrals (III) and the Perron type integrals (III) ${ }^{\prime}$ are always equivalent. Surprisingly, some descriptive integrals (II) are contained in some Perron type integrals $(\mathrm{I})^{\prime}$. It seems that the $\mathcal{L} P G$ integral cannot be characterized nicely descriptively. However we identify two situations (see Definition 15) for which the $\mathcal{L} P G$ integral admits descriptive characterizations. 


\section{Preliminaries}

We denote by $m^{*}(X)$ the outer measure of the set $X$ and by $m(A)$ the Lebesgue measure of $A$, whenever $A \subset \mathbb{R}$ is Lebesgue measurable. $d(A, x)$ denotes the density of the set $A$ at the point $x$ ([1], p. 18). For the definitions of $V B$, $A C$ and $T_{2}$, see [19]. Let $\mathcal{C}$ denote the class of all continuous functions, $\mathcal{D}$ the class of all Darboux functions, $\mathcal{B}_{1}$ the functions in Baire class one, and $\mathcal{B}$ or the collection of all Borel measurable functions. For two classes $\mathcal{A}_{1}, \mathcal{A}_{2}$ of real functions on a set $P$ let

$$
\mathcal{A}_{1} \boxplus \mathcal{A}_{2}=\left\{\alpha_{1} F_{1}+\alpha_{2} F_{2}: F_{1} \in \mathcal{A}_{1}, F_{2} \in \mathcal{A}_{2}, \alpha_{1}, \alpha_{2} \geq 0\right\} .
$$

Definition 1. Let $F:[a, b] \rightarrow \mathbb{R}$, and let $P$ be a closed subset of $[a, b]$, $c=\inf (P), d=\sup (P)$. Let $\left\{\left(c_{k}, d_{k}\right)\right\}_{k}$ be the intervals contiguous to $P$ and define $F_{P}:[c, d] \rightarrow \mathbb{R}$ by

$$
F_{P}(x)= \begin{cases}F(x) & \text { if } x \in P \\ \text { linear } & \text { on each }\left[c_{k}, d_{k}\right] .\end{cases}
$$

Definition 2. Let $F: P \rightarrow \mathbb{R}$ and $[a, b] \subseteq P$. Define $F_{a, b}: \mathbb{R} \rightarrow \mathbb{R}$ by

$$
F_{a, b}(x)= \begin{cases}F(a) & \text { if } x<a \\ F(x) & \text { if } x \in[a, b] \\ F(b) & \text { if } x>b .\end{cases}
$$

Definition 3. ([2]). A function $F: \mathbb{R} \rightarrow \mathbb{R}$ is said to be $\mathcal{D}_{-}$if $[F(\beta), F(\alpha)] \subseteq$ $F([\alpha, \beta])$, whenever $\alpha<\beta$ and $F(\beta)<F(\alpha)$. Clearly $F$ is $\mathcal{D}$ (Darboux) if $F$ and $-F$ are both $\mathcal{D}_{-}$.

Definition 4 (C. M. Lee). ([11]). A function $F: \mathbb{R} \rightarrow \mathbb{R}$ is said to be $u C M$ if $F$ is increasing on $[c, d]$ whenever $F$ is increasing on $(c, d)$. $F$ is said to be $C M$ if $F$ and $-F$ are both $u C M$.

Definition 5 (Garg). ([6]). Let $E \subseteq \mathbb{R}$ and $F: E \rightarrow \mathbb{R}$. The function $F$ is said to be lower internal if

$$
\liminf _{\substack{y \rightarrow x \\ y<x}} F(y) \leq F(x) \leq \limsup _{\substack{y \rightarrow x \\ y>x}} F(y)(\forall) x \in E .
$$

$F$ is said to be internal if $F$ and $-F$ are both lower internal. $F:[a, b] \rightarrow \mathbb{R}$ is said to be lower internal if $F_{a, b}$ is lower internal.

Definition 6. Let $P \subseteq[a, b], x_{0} \in P$ and $F: P \rightarrow \mathbb{R}$. 
- $F$ is said to be $\mathcal{C}_{i}$ at $x_{0}$ if $\lim \sup _{x} \nearrow_{x} F(x) \leq F\left(x_{0}\right)$, whenever $x_{0}$ is a left accumulation point for $P$, and $\underset{x \in P}{ } F\left(x_{0}\right) \leq \liminf _{x \rightarrow x_{0}} F(x)$, whenever $x_{0}$ is a right accumulation point for $P$.

- $F$ is said to be $\mathcal{C}_{i}^{*}$ at $x_{0}$ if $\lim _{\substack{x \nearrow_{x \in P} \\ x \in P}} F(x)$ exists and is finite, with $\lim _{x \succ x_{0}} F(x) \leq F\left(x_{0}\right)$ whenever $x_{0}$ is a left accumulation point for $F$, and if $\lim _{x \backslash x_{0}} F(x)$ exists and is finite, with $F\left(x_{0}\right) \leq \lim _{x \in P} \searrow_{x \in P} F(x)$ whenever $x_{0}$ is a right accumulation point for $F$.

- $F$ is said to be $\mathcal{C}_{i}$ (respectively $\mathcal{C}_{i}^{*}$ ) on $P$, if $F$ is so at each point $x \in P$.

Definition 7. Let $E \subset \mathbb{R}$ be an open set and $F: E \rightarrow \mathbb{R}$.

- $F$ is said to be $\mathcal{C}_{i, a p}$ at $x_{0} \in E$ if there exists a measurable set $E_{x_{0}} \subset E$ with $d\left(E_{x_{0}}, x_{0}\right)=1$ such that

$$
\limsup _{\substack{x \rightarrow x_{0} \\ x<x_{0}, x \in E_{x_{0}}}} F(x) \leq F\left(x_{0}\right) \leq \liminf _{\substack{x \rightarrow x_{0} \\ x>x_{0}, x \in E_{x_{0}}}} F(x) .
$$

- $F$ is said to be $\mathcal{C}_{i, a p}^{*}$ at $x_{0} \in E$ if there exists a measurable set $E_{x_{0}} \subset E$ with $d\left(E_{x_{0}}, x_{0}\right)=1$ such that the two limits

$$
\lim _{\substack{x \rightarrow x_{0} \\ x<x_{0}, x \in E_{x_{0}}}} F(x) \text { and } \lim _{\substack{x \rightarrow x_{0} \\ x>x_{0}, x \in E_{x_{0}}}} F(x)
$$

exist, are finite and

$$
\lim _{\substack{x \rightarrow x_{0} \\ x<x_{0}, x \in E_{x_{0}}}} F(x) \leq F\left(x_{0}\right) \leq \lim _{\substack{x \rightarrow x_{0} \\ x>x_{0}, x \in E_{x_{0}}}} F(x) .
$$

- The function $F$ is said to be $C_{i, a p}$ (respectively $\mathcal{C}_{i, a p}^{*}$ ) on a subset $A$ of $E$, if it is so at each point of $A$.

- Let $\mathcal{C}_{i, a p}[a, b]=\left\{F:[a, b] \rightarrow \mathbb{R}: F_{a, b}\right.$ is $\mathcal{C}_{i, a p}$ on $\left.\mathbb{R}\right\}$ and $\mathcal{C}_{i, a p}^{*}[a, b]=\{F$ : $[a, b] \rightarrow \mathbb{R}: F_{a, b}$ is $\mathcal{C}_{i, a p}^{*}$ on $\left.\mathbb{R}\right\}$.

Proposition 1. Let Inc $=\{F: \mathbb{R} \rightarrow \mathbb{R}: F$ is increasing $\}$.

(i) Let $E \subset \mathbb{R}$ be an open set and let $F_{n}, F: E \rightarrow \mathbb{R}, n=1,2, \ldots$. If each $F_{n} \in \mathcal{C}_{i, a p}$ on $E$ and $F_{n} \rightarrow F\left[\right.$ unif] on $E$, then $F \in \mathcal{C}_{i, a p}$ on $E$.

(ii) (i) remains true if $\mathcal{C}_{i, a p}$ is replaced by $\mathcal{C}_{i}$.

(iii) $\mathcal{C} \subset \mathcal{C} \boxplus$ Inc $\subset \mathcal{C}_{i}^{*} \subset \mathcal{C}_{i} \subset \mathcal{D}_{-} \mathcal{B}_{1} \subset \mathcal{D}_{-} \subset$ lower internal $\subset u C M$ on $[a, b]$. 
(iv) $\mathcal{C}_{a p} \subset \mathcal{C}_{a p} \boxplus I n c \subset \mathcal{C}_{i, a p}^{*} \subset \mathcal{C}_{i, a p} \subset$ lower internal $\subset u C M$ on $[a, b]$.

(v) $\mathcal{C}=\mathcal{C}_{i} \cap\left(-\mathcal{C}_{i}\right)$ and $\mathcal{C}_{a p}=\mathcal{C}_{i, a p} \cap\left(-\mathcal{C}_{i, a p}\right)$ on $[a, b]$.

(vi) $\mathcal{C}_{i, a p} \cap \mathcal{B}_{1} \subset \mathcal{D}_{-} \mathcal{B}_{1}=$ lower internal $\cap \mathcal{B}_{1} \subset u C M \cap \mathcal{B}_{1}$ on $[a, b]$.

Proof. (i) Let $\epsilon>0$ and choose $N$ large enough such that

$$
\left|F_{n}(x)-F(x)\right|<\frac{\epsilon}{3} \quad(\forall) x \in E, \text { whenever } n \geq N .
$$

Let $x_{0} \in E$. Since $F_{N} \in \mathcal{C}_{i, a p}$ at $x_{0}$, there exist a measurable set $E_{x_{0}}$ with $d\left(E_{x_{0}}, x_{0}\right)=1$ and a $\delta>0$ such that

$$
F_{N}(u)-\frac{\epsilon}{3}<F_{N}\left(x_{0}\right)<F_{N}(v)+\frac{\epsilon}{3},
$$

whenever $u \in\left(x_{0}-\delta, x_{0}\right] \cap E_{x_{0}}$ and $v \in\left[x_{0}, x_{0}+\delta\right) \cap E_{x_{0}}$. Then

$$
F\left(x_{0}\right)<F_{N}\left(x_{0}\right)+\frac{\epsilon}{3}<F_{N}(v)+\frac{2 \epsilon}{3}<F(v)+\epsilon
$$

and

$$
F\left(x_{0}\right)>F_{N}\left(x_{0}\right)-\frac{\epsilon}{3}>F_{N}(u)-\frac{2 \epsilon}{3}>F(u)-\epsilon .
$$

Therefore $F \in \mathcal{C}_{i, a p}$ at $x_{0}$.

(ii) See [2], p. 32.

(iii) and (iv) These follow from definitions and Theorem 2.4.2, (vi) of [2].

(v) This follows by the definitions.

(vi) The first inclusion and the equality follow by (iv) and Theorem 2.5.1, (i), (iv) of [2]. For the last inclusion see Theorem 2.4.2, (viii) of [2].

Definition 8. A function $F: P \rightarrow \mathbb{R}$ is said to be $\underline{L}$ on $P$ if there exists a real constant $\alpha$ such that $F(y)-F(x) \geq \alpha(y-x)$, whenever $x, y \in P, x<y$. Clearly $F$ is Lipschitz (abbreviated $(L)$ ) on $P$ if and only if $F$ and $-F$ are both $\underline{L}$ on $P$.

Definition 9. ([14], p. 236). A function $F: P \rightarrow \mathbb{R}$ is said to be $\underline{A C}$ (respectively $\overline{A C}$ ) if for every $\epsilon>0$ there is a $\delta>0$ such that

$$
\sum_{k=1}^{n}\left(F\left(b_{k}\right)-F\left(a_{k}\right)\right)>-\epsilon \quad\left(\text { respectively } \sum_{k=1}^{n}\left(F\left(b_{k}\right)-F\left(a_{k}\right)\right)<\epsilon\right),
$$

whenever $\left\{\left[a_{k}, b_{k}\right]\right\}, k=1,2, \ldots, n$ is a finite set of nonoverlapping closed

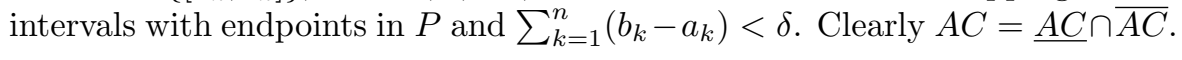


Definition 10. A function $F: P \rightarrow \mathbb{R}$ is said to be $V B G$ (respectively $A C G$, $\underline{A C G}, \overline{A C} G, \underline{L} G, L G,(A C \cap \underline{L}) G)$ on $P$ if there exists a sequence of sets $\left\{P_{n}\right\}$ with $P=\cup_{n} P_{n}$, such that $F$ is $V B$ (respectively $A C, \underline{A C}, \overline{A C}, \underline{L}, L, A C \cap \underline{L}$ ) on each $P_{n}$. If in addition the sets $P_{n}$ are supposed to be closed we obtain the classes $[V B G],[A C G],[\underline{A C G}],[\overline{A C G}],[\underline{L} G],[(A C \cap \underline{L}) G]$. Note that condition $A C G$ used here differs from that of [19] (because in our definition the continuity is not assumed).

Definition 11. Let $F:[a, b] \rightarrow \mathbb{R}$ and $P \subseteq[a, b]$.

- $F$ is said to satisfy Lusin's condition $(N)$ on $P$ if $m^{*}(F(Z))=0$ whenever $Z$ is a null subset of $P([19]$, p. 224).

- $F$ is said to satisfy Saks' condition $N^{+\infty}$ on $P$ if the set $F(\{x \in P$ : $\left.\left.\left(F_{\mid P}\right)^{\prime}(x)=+\infty\right\}\right)$ is of measure zero. $F$ is said to be $N^{-\infty}$ on $P$ if $-F$ is $N^{+\infty}$ on $P$, i.e., the set $F\left(\left\{x \in P:\left(F_{\mid P}\right)^{\prime}(x)=-\infty\right\}\right)$ is of measure zero.

Definition 12. Let $F:[a, b] \rightarrow \mathbb{R}, P \subset[a, b] . F$ is said to be $\underline{M}$ on $P$ if $F \in \underline{A C}$ on $Q$, whenever $Q=\bar{Q} \subset P$ and $F \in V B \cap \mathcal{C}$ on $Q$.

A function $F$ is said to satisfy Foran's condition $(M)$ on $P$ if $F$ is simultaneously $\underline{M}$ and $\bar{M}$ (i.e., $F$ is $A C$ on $Q$ whenever $Q$ is a closed subset of $P$ and $F \in \overline{V B} \cap \mathcal{C}$ on $Q$, see [5]).

Definition 13. ([2], p. 6). Let $F:[a, b] \rightarrow \mathbb{R}, P \subseteq[a, b]$. Put

- $\mathcal{O}^{\infty}(F ; P)=\inf \left\{\sum_{i=1}^{\infty} \mathcal{O}\left(F ; P_{i}\right): \cup_{i=1}^{\infty} P_{i}=P\right\}$;

- $\mathcal{O}_{+}^{\infty}(F ; P)=\inf \left\{\sum_{i=1}^{\infty} \mathcal{O}_{+}\left(F ; P_{i}\right): \cup_{i=1}^{\infty} P_{i}=P\right\}$;

- $\mathcal{O}_{-}^{\infty}(F ; P)=\sup \left\{\sum_{i=1}^{\infty} \mathcal{O}_{-}\left(F ; P_{i}\right): \cup_{i=1}^{\infty} P_{i}=P\right\}$;

Definition 14. ([2], p. 78). Let $F:[a, b] \rightarrow \mathbb{R}, P \subseteq[a, b] . F$ is said to be $(\bar{N})$ on $P$ if $\mathcal{O}_{+}^{\infty}(F ; Z)=0$, whenever $Z \subset P$ and $m(Z)=0$. F is said to be $(\underline{N})$ on $P$ if $-F$ is $(\bar{N})$ on $P$; i.e., $\mathcal{O}_{-}^{\infty}(F ; Z)=0$.

Theorem A. Let $F:[a, b] \rightarrow \mathbb{R}$.

a) (C. M. Lee) If $F \in u C M \cap[\underline{A C G}]$ on $[a, b]$, then $F$ is increasing on $[a, b]$ if and only if $F^{\prime}(x) \geq 0$ a.e. where $F$ is derivable on $[a, b]$.

b) (C. M. Lee) If $F \in \mathcal{D B}_{1} \cap(N)$ on $[a, b]$ and $F^{\prime}(x) \geq 0$ a.e. where $F$ is derivable, then $F$ is increasing and continuous on $[a, b]$.

c) If $F \in \mathcal{D}_{-} \mathcal{B}_{1} T_{2} \cap(\underline{N})$ on $[a, b]$, and $F^{\prime}(x) \geq 0$ a.e. where $F$ is derivable on $[a, b]$, then $F$ is increasing on $[a, b]$. In particular, the assertion remains true if $T_{2}$ is replaced by $V B G$. 
d) $(\underline{N}) \cap V B G \cap \mathcal{B}$ or $=\underline{M} \cap V B G \cap \mathcal{B}$ or is a real upper linear space on a Borel measurable subset of $[a, b]$.

e) $[V B G] \cap(\underline{N})=[V B G] \cap \underline{M}$ is a real upper linear space on a closed subset of $[a, b]$.

f) $V B G \cap(N) \cap \mathcal{B}$ or $=V B G \cap(M) \cap \mathcal{B}$ or is a linear space on a Borel subset of $[a, b]$.

g) $[V B G] \cap(N)=[V B G] \cap(M)$ is a linear space on a closed subset of $[a, b]$.

h) $V B \cap(N)=V B \cap(M)$ is a real space on $[a, b]$.

i) $V B \cap(\underline{N})=V B \cap \underline{M}$ is an upper semilinear space on $[a, b]$.

Proof. a) See Theorem 1 of [11], p. 70.)

b) See Theorem 1 of [12], p. 61, or Corollary 4.3 .4 of [2].)

c) By Corollary 4.3.1 of [2], if $F \in \mathcal{D}_{-} \mathcal{B}_{1} T_{2} \cap N^{-\infty}$ on $[a, b]$, and $F^{\prime}(x) \geq 0$ a.e. where $F$ is derivable on $[a, b]$, then $F$ is increasing on $[a, b]$. But $(\underline{N}) \subset$ $N^{-\infty}$ (see Lemma 2.21.1 of [2]).

For the second part, $V B G \subset T_{2}$ follows from [19], p. 279 .

d) See Theorem 2 of [4].

e) See Theorem 1 of [4].

f) See Theorem 2, (ii) of [4].

g) Theorem 1, (ii) of [4].

h) and i) follow by Lemma 2 of [4].

Remark 1. 1) A generalization of Theorem A, a) can be found in [2] (see Corollary 4.3.5).

2) Theorem A, b) was proved by C. M. Lee using Bruckner's Theorem 2.2 of [1] and the Banach-Ellis Theorem (see Theorem 2 of [2], p. 187, or Theorem B of [12], p. 62).

We state the following generalization of Theorem A, b) (see Corollary 4.3.3 of $[2]):$ Let $F:[a, b] \rightarrow \mathbb{R}, F \in \mathcal{D}_{-} \mathcal{B}_{1} \cap(N)$ on $[a, b]$. If $F^{\prime}(x) \geq 0$ a.e. where $F$ is derivable, then $F$ is increasing on $[a, b]$.

3) Theorem A, c) is an extension of Theorem A, b) and Corollary 4.3.3 mentioned above.

4) Theorem A, e) is a special case of Theorem A, d).

5) That $[V B G] \cap(N)$ is a linear space on a closed subset of $[a, b]$ was first proved by Sarkhel and Kar in [23] (see also [3]).

6) That $V B \cap(N)$ is a real space on $[a, b]$ was first proved by Sarkhel and Kar in $[23]$. 


\section{Classes of the First and the Second Type. The condition $((*))$. Examples}

Definition 15. Let $\mathcal{A} \subset\{F: \mathbb{R} \rightarrow \mathbb{R}\}$.

- The class $\mathcal{A}$ is said to satisfy the condition $(*)$ if $F_{a, b} \in \mathcal{A}$ for $[a, b] \subset \mathbb{R}$ whenever $F \in \mathcal{A}$.

- $\mathcal{A}$ is said to be of the first type if $F_{[[c, d]}$ is continuous on $[c, d]$ whenever $F \in \mathcal{A}$ and $F$ is increasing on $[c, d]$.

- $\mathcal{A}$ is said to be of the second type if $F \in \mathcal{A}$ whenever $F=F_{1}+F_{2}$, $F_{1}, F_{2}: \mathbb{R} \rightarrow \mathbb{R}, F_{1} \in \mathcal{A}$ and $F_{2}$ is increasing.

Theorem 1 (Examples).

(i) Any class $\mathcal{A} \subset\{F: \mathbb{R} \rightarrow \mathbb{R}: F$ is internal $\}$ is of the first type.

(ii) The following classes satisfy the property (*).

- $\left\{F: \mathbb{R} \rightarrow \mathbb{R}: F \in \mathcal{D}_{-}\right\}$;

- $\{F: \mathbb{R} \rightarrow \mathbb{R}: F \in \mathcal{D}\}$;

- $\{F: \mathbb{R} \rightarrow \mathbb{R}: F \in u C M\}$;

- $\{F: \mathbb{R} \rightarrow \mathbb{R}: F \in C M\}$;

- $\{F: \mathbb{R} \rightarrow \mathbb{R}: F \in$ lower internal $\}$;

- $\{F: \mathbb{R} \rightarrow \mathbb{R}: F \in$ internal $\}$;

(iii) The following classes satisfy the property (*), are linear spaces and of the first type.

- $\mathcal{C}=\{F: \mathbb{R} \rightarrow \mathbb{R}: F$ is continuous on $\mathbb{R}\} ;$

- $\mathcal{C}_{a p}=\{F: \mathbb{R} \rightarrow \mathbb{R}: F$ is approximately continuous on $\mathbb{R}\}$;

(iv) The following classes satisfy the property (*), are upper semilinear spaces and of the second type.

- Inc $=\{F: \mathbb{R} \rightarrow \mathbb{R}: F$ is increasing $\}$;

- $\mathcal{C} \boxplus$ Inc and $\mathcal{C}_{a p} \boxplus$ Inc;

- $\mathcal{C}_{i}^{*}=\left\{F: \mathbb{R} \rightarrow \mathbb{R}: F\right.$ is $\mathcal{C}_{i}^{*}$ on $\left.\mathbb{R}\right\}$;

- $\mathcal{C}_{i}=\left\{F: \mathbb{R} \rightarrow \mathbb{R}: F\right.$ is $\mathcal{C}_{i}$ on $\left.\mathbb{R}\right\}$;

- $\mathcal{C}_{i, a p}^{*}=\left\{F: \mathbb{R} \rightarrow \mathbb{R}: F\right.$ is $\mathcal{C}_{i, a p}^{*}$ on $\left.\mathbb{R}\right\}$;

- $\mathcal{C}_{i, a p}=\left\{F: \mathbb{R} \rightarrow \mathbb{R}: F\right.$ is $\mathcal{C}_{i, a p}$ on $\left.\mathbb{R}\right\}$; 
Proof. (i) Let $[c, d] \subset \mathbb{R}$ such that $F$ is increasing on $[c, d]$, and let $x_{0} \in[c, d)$. Since $F$ is increasing, we have that $F\left(x_{0}\right) \leq \lim _{\substack{y>x_{0} \\ y>x_{0}}} F(y)$. But we also have

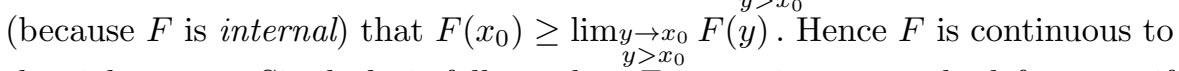
the right at $x_{0}$. Similarly it follows that $F$ is continuous to the left at $x_{0}$, if $x_{0} \in(c, d]$. Therefore $F_{\mid[c, d]}$ is continuous on $[c, d]$.

The other assertions are evident.

\section{The Saltus of a Function}

Lemma 1. Let $F:[a, b] \rightarrow \mathbb{R}$ be $a V B$ function and $A \subset(a, b)$ be the set of all interior discontinuity points of $F$. Let $H:[a, b] \rightarrow \mathbb{R}$,

$$
H(x)= \begin{cases}0 & \text { if } x=a \\ V(F ;[a, x]) & \text { if } x \in(a, b]\end{cases}
$$

and let $h(x)=H(x)-F(x)$ for every $x \in[a, b]$. Then we have

(i) A is at most countable and the following limits exist.

$$
F(x+)=\lim _{\substack{y \rightarrow x \\ y>x}} F(y), x \in A \cup\{a\} \text { and } F(x-)=\lim _{\substack{y \rightarrow x \\ y<x}} F(y), x \in A \cup\{b\} .
$$

(ii) $H, h$ are increasing on $[a, b]$, and $H, h$ are continuous on $(a, b) \backslash A$.

Proof. (i) See Corollary 2 of [13], p. 219.

(ii) See Theorem 6 and Theorem 1 of [13], pp. 218, 223.

Definition 16 (The Saltus of an Increasing Function). ([13], p. 206).

Let $F:[a, b] \rightarrow \mathbb{R}$ be an increasing function, and let $A=\left\{a_{1}, a_{2}, \ldots\right\} \subset(a, b)$ be a countable set that contains all interior discontinuity points of $F$ (see Theorem 1 of [13], p. 205). Let $s_{F}:[a, b] \rightarrow \mathbb{R}, s_{F}(a)=0$ and for every $x \in(a, b]$,

$$
s_{F}(x)=f(a+)-f(a)+\sum_{t \in(a, x) \cap A}(f(t+)-f(t-))+f(x)-f(x-) .
$$

Clearly $F(t+)$ and $F(t-)$ exist and $s_{F}(b) \leq F(b)-F(a)$ (see Theorem 1 of [13], p. 205). Clearly $s_{F}$ is an increasing function, and it is called the saltus of the function $F$.

Lemma 2. (Theorem 2 of [13], p. 206). Let $F$ and $s_{F}$ be as in Definition 16. Then $F-s_{F}$ is increasing and continuous on $[a, b]$. 
Definition 17 (The Saltus of a VB function). Let $F, H, h:[a, b] \rightarrow \mathbb{R}$ and $A=\left\{a_{1}, a_{2}, \ldots\right\} \subset(a, b)$ be as in Lemma 1 . Let $s_{F}:[a, b] \rightarrow \mathbb{R}, s_{F}(a)=0$ and for every $x \in(a, b]$,

$$
\begin{gathered}
s_{F}(x)=s_{H}(x)-s_{h}(x)= \\
=F(a+)-F(a)+\sum_{t \in(a, x) \cap A}(F(t+)-F(t-))+F(x)-F(x-)
\end{gathered}
$$

(see [13], p. 219). $s_{F}$ is called the saltus of the function $F$.

Let $S_{F}:[a, b] \rightarrow \mathbb{R}, S_{F}(a)=0$, and for every $x \in(a, b]$,

$$
\begin{gathered}
S_{F}(x)=|F(a+)-F(a)|+ \\
+\sum_{t \in(a, x) \cap A}(|F(t+)-F(t)|+|F(t)-F(t-)|)+|F(x)-F(x-)| .
\end{gathered}
$$

(That the above series is convergent follows from the footnote on p. 235 of [13].)

For $A$ infinite, let $k$ be a positive integer. We may suppose without loss of generality that $a<a_{1}<a_{2}<\ldots<a_{k}<b$. Let $S_{F, k}:[a, b] \rightarrow \mathbb{R}$ be defined as follows.

$$
\begin{aligned}
& S_{F, k}(a)=0 ; \\
& S_{F, k}(x)=\sum_{t \in A \cap(a, x)}(|F(t+)-F(t)|+|F(t)-F(t-)|)+|F(x)-F(x-)| \\
& \text { if } x \in\left(a, a_{1}\right) \text {; } \\
& S_{F, k}(x)=\sum_{t \in A \cap\left(a, a_{1}\right)}(|F(t+)-F(t)|+|F(t)-F(t-)|)=: \alpha_{1} \\
& \text { if } x=a_{1} \text {; } \\
& S_{F, k}(x)=\alpha_{1}+\sum_{t \in A \cap\left(a_{1}, x\right)}(|F(t+)-F(t)|+|F(t)-F(t-)|)+|F(x)-F(x-)| \\
& \text { if } x \in\left(a_{1}, a_{2}\right) \text {; } \\
& S_{F, k}(x)=\alpha_{1}+\sum_{t \in A \cap\left(a_{1}, a_{2}\right)}(|F(t+)-F(t)|+|F(t)-F(t-)|)=: \alpha_{2} \\
& \text { if } x=a_{2} \text {; } \\
& S_{F, k}(x)=\alpha_{k-1}+\sum_{t \in A \cap\left(a_{k-1}, a_{k}\right)}(|F(t+)-F(t)|+|F(t)-F(t-)|)=: \alpha_{k} \\
& \text { if } x=a_{k} \text {; } \\
& S_{F, k}(x)=\alpha_{k}+\sum_{t \in A \cap\left(a_{k}, x\right)}(|F(t+)-F(t)|+|F(t)-F(t-)|)+|F(x)-F(x-)| \\
& \text { if } x \in\left(a_{k}, b\right) \text {; } \\
& \begin{array}{l}
S_{F, k}(x)=\alpha_{k}+\sum_{t \in A \cap\left(a_{k}, b\right)}(|F(t+)-F(t)|+|F(t)-F(t-)|)=: \alpha_{k+1} \\
\quad \text { if } x=b .
\end{array}
\end{aligned}
$$


Lemma 3. ([13], p. 220). Let $F:[a, b] \rightarrow \mathbb{R}$ be a $V B$ function. Then $F-s_{F}$ is continuous on $[a, b]$.

Lemma 4. For $F, s_{F}, S_{F}$ and $S_{F, k}$ defined above we have.

(i) $s_{F} \in(N)$ on $[a, b]$ and $s_{F}^{\prime}(x)=0$ a.e. on $[a, b]$.

(ii) If $F \in(M)$ on $[a, b]$, then $F-s_{F} \in A C$ on $[a, b]$. Particularly, the assertion remains true for $F \in(N)$.

(iii) If $F \in \underline{M}$ on $[a, b]$, then $F-s_{F} \in \underline{A C}$ on $[a, b]$. Particularly, the assertion remains true for $F \in(\underline{N})$.

(iv) $S_{F}, S_{F, k}$ are increasing and $(N)$ on $[a, b]$. Moreover, $S_{F}^{\prime}=S_{F, k}^{\prime}=0$ a.e. on $[a, b]$.

(v) $S_{F, k}(b)=\sum_{i=k+1}^{\infty}\left(\left|F\left(a_{i}+\right)-F\left(a_{i}\right)\right|+\left|F\left(a_{i}\right)-F\left(a_{i}-\right)\right|\right) \searrow 0$, for $k \rightarrow \infty$.

(vi) $s_{F}+S_{F}$ is increasing and $s_{F}-S_{F}$ is decreasing on $[a, b] ; s_{F}+S_{F, k}$ is increasing and $s_{F}-S_{F, k}$ is decreasing on each component of the open set $(a, b) \backslash\left\{a_{1}, a_{2}, \ldots, a_{k}\right\} ;$ hence $s_{F}+S_{F, k} \in[\underline{A C G}]$ and $s_{F}-S_{F, k} \in[\overline{A C G}]$ on $[a, b]$.

(vii) If $F \in(N)$ on $[a, b]$, then $F+S_{F, k} \in[\underline{A C G}]$ and $F-S_{F, k} \in[\overline{A C} G]$ on $[a, b]$.

Proof. (i) To show that $s_{F} \in(N)$, we shall use Sarkhel and Kar's technique of [23] (see Corollary 3.6.1). From Definition 16 it follows that $s_{F}=s_{H}-s_{h}$. We show that $s_{H} \in(N)$ on $[a, b]$. But

$$
\begin{gathered}
s_{H}([a, b]) \subseteq\left[0, s_{H}(b)\right] \backslash\left(\left(0, s_{H}(a+)\right) \cup\left(s_{H}(b-), s_{H}(b)\right) \cup\right. \\
\cup\left(\cup_{k=1}^{\infty}\left(\left(s_{H}\left(a_{k}-\right), s_{H}\left(a_{k}\right)\right) \cup\left(s_{H}\left(a_{k}\right), s_{H}\left(a_{k}+\right)\right)\right)\right)
\end{gathered}
$$

and $s_{H}(a+)=H(a+)-H(a)$. Also, for each $k=1,2, \ldots$,

$$
\begin{gathered}
s_{H}\left(a_{k}+\right)-s_{H}\left(a_{k}\right)=H\left(a_{k}+\right)-H\left(a_{k}\right), \\
s_{H}\left(a_{k}\right)-s_{H}\left(a_{k}-\right)=H\left(a_{k}\right)-H\left(a_{k}-\right)
\end{gathered}
$$

and $s_{H}(b)-s_{H}(b-)=H(b)-H(b-)$. Since

$$
s_{H}(b)=H(a+)-H(a)+\sum_{k=1}^{\infty}\left(H\left(a_{k}+\right)-H\left(a_{k}-\right)\right)+H(b)-H(b-),
$$

it follows that $m\left(s_{H}([a, b])\right)=0$. Hence $s_{H} \in(N)$ on $[a, b]$. Of course $s_{h} \in(N)$ on $[a, b]$. Since $s_{H}, s_{h} \in V B$ on $[a, b]$, by Theorem A, h), it follows that 
$s_{F} \in(N)$ on $[a, b]$. Clearly, $s_{H}$ is derivable a.e. on $[a, b]$. By Krzyzewski's Lemma (see for example [2], p. 70), we obtain that $s_{H}^{\prime}=0$ a.e. on $[a, b]$. Therefore $s_{F}^{\prime}=s_{H}^{\prime}-s_{h}^{\prime}=0$ a.e. on $[a, b]$.

(ii) From (i), Theorem A, h) and Lemma 3, we obtain that $F-s_{F} \in$ $V B \cap \mathcal{C} \cap(N)=A C$ on $[a, b]$ (see the Banach-Zarecki Theorem).

(iii) Again from (i), Theorem A, i) and Lemma 3, it follows that $F-s_{F} \in$ $V B \cap \mathcal{C} \cap \underline{M} \subset \underline{A C}$ (see the definition of $\underline{M}$ ).

(iv) Let $a \leq x_{1}<x_{2} \leq b$. Then

$$
\begin{gathered}
S_{F}\left(x_{2}\right)-S_{F}\left(x_{1}\right)=\left|F\left(x_{1}+\right)-F\left(x_{1}\right)\right|+ \\
+\sum_{t \in\left(x_{1}, x_{2}\right) \cap A}(|F(t+)-F(t)|+|F(t)-F(t-)|)+\left|F\left(x_{2}\right)-F\left(x_{2}-\right)\right|,
\end{gathered}
$$

so $S_{F}$ is increasing on $[a, b]$. Let $\left[x_{1}, x_{2}\right]$ be contained in one of the following closed intervals: $\left[a, a_{1}\right],\left[a_{1}, a_{2}\right], \ldots,\left[a_{k-1}, a_{k}\right],\left[a_{k}, b\right]$. Then

$$
S_{F, k}\left(x_{2}\right)-S_{F, k}\left(x_{1}\right) \geq \sum_{t \in\left(x_{1}, x_{2}\right) \cap A}(|F(t+)-F(t)|+|F(t)-F(t-)|),
$$

so $S_{F, k}$ is increasing on each $\left[a, a_{1}\right],\left[a_{1}, a_{2}\right], \ldots,\left[a_{k-1}, a_{k}\right],\left[a_{k}, b\right]$. Therefore $S_{F, k}$ is increasing on $[a, b]$.

We have

$$
\begin{gathered}
S_{F}(b)=|F(a+)-F(a)|+\sum_{t \in(a, b)}(|F(t+)-F(t)|+|F(t)-F(t-)|)+|F(b)-F(b-)| ; \\
S_{F}(y)-S_{F}(x)=|F(x+)-F(x)|+ \\
+\sum_{t \in(x, y) \cap A}(|F(t+)-F(t)|+|F(t)-F(t-)|)+|F(y)-F(y-)| ; \\
S_{F}\left(a_{i}+\right)-S_{F}\left(a_{i}\right)=\left|F\left(a_{i}+\right)-F\left(a_{i}\right)\right| ; \\
S_{F}(a+)-S_{F}(a)=|F(a+)-F(a)| ; \\
S_{F}\left(a_{i}\right)-S_{F}\left(a_{i}-\right)=\left|F\left(a_{i}\right)-F\left(a_{i}-\right)\right| ; \\
S_{F}(b)-S_{F}(b-)=|F(b)-F(b-)| ; \\
S_{F}(b)=\left[0, S_{F}(b)\right] \backslash\left(\left(0, S_{F}(a+)\right) \cup\left(S_{F}(b-), S_{F}(b)\right) \cup\right. \\
\left.\cup\left(\cup_{i=1}^{\infty}\left(S_{F}\left(a_{i}-\right), S_{F}\left(a_{i}\right)\right) \cup\left(S_{F}\left(a_{i}\right), S_{F}\left(a_{i}+\right)\right)\right)\right) .
\end{gathered}
$$

Therefore $m^{*}\left(S_{F}([a, b])\right)=0$; so $S_{F} \in(N)$ on $[a, b]$. By Krzyzewski's Lemma (see for example [2], p. 70$), S_{F}^{\prime}(x)=0$ a.e. on $[a, b]$. Similarly it follows that 
$S_{F, k} \in(N)$ and $S_{F, k}^{\prime}(x)=0$ a.e. on each $\left[a, a_{1}\right],\left[a_{1}, a_{2}\right], \ldots,\left[a_{k-1}, a_{k}\right],\left[a_{k}, b\right]$. Hence $S_{F, k} \in(N)$ on $[a, b]$ and $S_{F, k}^{\prime}(x)=0$ a.e. on $[a, b]$.

(v) This is obvious.

(vi) Let $a \leq x<y \leq b$. Then

$$
s_{F}(y)-s_{F}(x)=F(x+)-F(x)+\sum_{t \in(x, y) \cap A}(F(t+)-F(t))+F(y)-F(y-)
$$

and

$$
\begin{gathered}
S_{F}(y)-S_{F}(x)=|F(x+)-F(x)|+ \\
+\sum_{t \in(x, y) \cap A}(|F(t+)-F(t)|+|F(t)-F(t-)|)+|F(y)-F(y-)| .
\end{gathered}
$$

Therefore

$$
\left(s_{F}+S_{F}\right)(y)-\left(s_{F}+S_{F}\right)(x) \geq 0
$$

and

$$
\left(s_{F}-S_{F}\right)(y)-\left(s_{F}-S_{F}\right)(x) \leq 0,
$$

hence $s_{F}+S_{F}$ is increasing on $[a, b]$ and $s_{F}-S_{F}$ is decreasing on $[a, b]$.

Let $\left[x_{1}, x_{2}\right] \subset\left(a, a_{1}\right) \cup\left(a_{1}, a_{2}\right) \cup \ldots \cup\left(a_{k-1}, a_{k}\right) \cup\left(a_{k}, b\right)$. Then

$s_{F}\left(x_{2}\right)-s_{F}\left(x_{1}\right)=F\left(x_{1}+\right)-F\left(x_{1}\right)+\sum_{t \in\left(x_{1}, x_{2}\right) \cap A}(F(t+)-F(t-))+F\left(x_{2}\right)-F\left(x_{2}-\right)$

and

$$
\begin{gathered}
S_{F, k}\left(x_{2}\right)-S_{F, k}\left(x_{1}\right)=\left|F\left(x_{1}+\right)-F\left(x_{1}\right)\right|+ \\
+\sum_{t \in\left(x_{1}, x_{2}\right) \cap A}(|F(t+)-F(t)|+|F(t)-F(t-)|)+\left|F\left(x_{2}\right)-F\left(x_{1}\right)\right| .
\end{gathered}
$$

Therefore

$\left(s_{F}+S_{F, k}\right)\left(x_{2}\right)-\left(s_{F}+S_{F, k}\right)\left(x_{1}\right)=\left(s_{F}\left(x_{2}\right)-s_{F}\left(x_{1}\right)\right)+\left(S_{F, k}\left(x_{2}\right)-S_{F, k}\left(x_{1}\right)\right) \geq 0$

and

$\left(s_{F}-S_{F, k}\right)\left(x_{2}\right)-\left(s_{F}-S_{F, k}\right)\left(x_{1}\right)=\left(s_{F}\left(x_{2}\right)-s_{F}\left(x_{1}\right)\right)-\left(S_{F, k}\left(x_{2}\right)-S_{F, k}\left(x_{1}\right)\right) \leq 0$.

It follows that $s_{F}+S_{F, k}$ is increasing and $s_{F}-S_{F, k}$ is decreasing on each component interval of the open set $\left(a, a_{1}\right) \cup\left(a_{1}, a_{2}\right) \cup \ldots \cup\left(a_{k-1}, a_{k}\right) \cup\left(a_{k}, b\right)$.

(vii) From (ii) and (vi) it follows that

$$
F+S_{F, k}=\left(F-s_{F}\right)+s_{F}+S_{F, k} \in[\underline{A C G}] \text { on }[a, b]
$$

and

$$
F-S_{F, k}=\left(F-s_{F}\right)+s_{F}-S_{F, k} \in[\overline{A C} G] \text { on }[a, b] .
$$


Remark 2. That $s_{H}^{\prime}=0$ a.e. in Lemma 4 , (i), and that $S_{F}^{\prime}=S_{F, k}^{\prime}=0$ a.e. in Lemma 4, (vi), can also be proved as follows. (See the proof of Corollary 3.6.1 of [23].) Take for example $s_{H}$. Clearly $s_{H}$ is derivable a.e. on $[a, b]$ and $s_{H}(x) \geq 0$ a.e. on $[a, b]$. Then by Theorem 12 of [24], it follows that

$$
(\mathcal{L}) \int_{a}^{b} s_{H}^{\prime}(t) d t=m^{*}\left(s_{H}([a, b])\right)=0 .
$$

By Theorem 6 of [13] (p. 188), it follows that $s_{H}^{\prime}(x)=0$ a.e on $[a, b]$.

Corollary 1 (A Jordan Type Theorem). Let $F:[a, b] \rightarrow \mathbb{R}, F \in V B \cap(N)$. Then there exist $H, h:[a, b] \rightarrow \mathbb{R}$ such that $F=H-h$ and $H, h$ are increasing and $(N)$ on $[a, b]$.

Proof. Let

$$
H=\frac{1}{2}\left(F+S_{F}+V_{F-s_{F}}\right)=\frac{1}{2}\left(\left(F-s_{F}\right)+s_{F}+S_{F}+V_{F-s_{F}}\right)
$$

and

$$
h=-\frac{1}{2}\left(F-S_{F}-V_{F-s_{F}}\right)=-\frac{1}{2}\left(\left(F-s_{F}\right)+s_{F}-S_{F}-V_{F-s_{F}}\right) .
$$

But $F-s_{F} \in A C$ (see Lemma 4, (ii)); so $V_{F-s_{F}} \in A C$ on $[a, b]$. Therefore $\left(F-s_{F}\right)+V_{F-s_{F}}$ is $A C$ and increasing on $[a, b]$, and $\left(F-s_{F}\right)-V_{F-s_{F}}$ is $A C$ and decreasing on $[a, b]$. From Lemma 4 , (vi), we obtain that $s_{F}+S_{F}$ is increasing and $s_{F}-S_{F}$ is decreasing on $[a, b]$. Therefore $H, h$ are increasing on $[a, b]$, and by Lemma 4 , (i), (vi) we obtain that $s_{F}, S_{F} \in(N)$ on $[a, b]$. It follows that $H, h \in(N)$ on $[a, b]$ (see Theorem A, h)).

Corollary 2. Let $F:[a, b] \rightarrow \mathbb{R}, F \in V B \cap(N)$. Then there exists a sequence $\left\{h_{k}\right\}_{k}$ of functions, $h_{k}:[a, b] \rightarrow \mathbb{R}$, having the following properties.

(i) $h_{k}(a)=0$ for each $k$;

(ii) $h_{k}$ is increasing on $[a, b]$ for each $k$;

(iii) $F+h_{k} \in[\underline{A C G}]$ and $F-h_{k} \in[\overline{A C G}]$ on $[a, b]$ for each $k$;

(iv) $\left\{h_{k}(b)\right\}_{k}$ is a decreasing sequence converging to 0 .

Proof. Put for example $h_{k}=S_{F, k}$. 


\section{Relations between $[\underline{\mathrm{ACG}}]$ and $[\underline{\mathrm{LG}}]$}

Lemma 5. Let $F:[a, b] \rightarrow \mathbb{R}, F \in[\underline{A C G}]$. For $\epsilon>0$ there exists a function $H:[a, b] \rightarrow \mathbb{R}$ with the following properties.

(i) $H(a)=0, H(b) \leq \epsilon$;

(ii) $H$ is increasing and $A C$ on $[a, b]$;

(iii) $F+H \in[\underline{L} G]$ on $[a, b]$.

(iv) If $F \in[A C G]$, then $F+H \in[(\underline{L} \cap A C) G]$ on $[a, b]$.

Proof. We may suppose without loss of generality that $F(a)=0$. Since $F \in[\underline{A C G}]$ on $[a, b]$, there exists a sequence $\left\{P_{n}\right\}_{n}$ of closed sets such that $[a, b]=\cup_{n} P_{n}$ and $F \in \underline{A C}$ on each $P_{n}$. We may suppose without loss of generality that each $P_{n}$ contains the points $a$ and $b$. Then $F_{P_{n}}:[a, b] \rightarrow \mathbb{R}$ is $A C$ on $[a, b]$ (see Theorem 2.11.1, (xvii) of [2]). By Theorem 2.14.5 of [2], there exist $F_{n}$ and $h_{n}$ such that $F_{P_{n}}=F_{n}+h_{n}, F_{n} \in A C$ on $[a, b], h_{n}(a)=0$, $h_{n}$ is singular and increasing on $[a, b]$. Clearly $\underline{D} F_{n}$ is summable on $[a, b]$ and

$$
(\mathcal{L}) \int_{a}^{x} \underline{D} F_{n}(t) d t=F_{n}(x)-F_{n}(a)=F_{n}(x)
$$

(see [13], vol. I, p. 255). For $\epsilon / 2^{n}$ there exists a function $u_{n}:(-\infty,+\infty]$ (see [13], vol. II, p. 166) such that $u_{n}$ is lower semicontinuous on $[a, b] ; u_{n}(x) \geq$ $\underline{D} F_{n}(x)$ on $[a, b] ; u_{n}$ is summable and

$$
(\mathcal{L}) \int_{a}^{b} u_{n}(t) d t<\frac{\epsilon}{2^{n}}+(\mathcal{L}) \int_{a}^{b} \underline{D} F_{n}(t) d t .
$$

Let $H_{n}(x)=(\mathcal{L}) \int_{a}^{x}\left(u_{n}(t)-\underline{D} F_{n}(t)\right) d t$. Then $H_{n}(a)=0, H_{n}(b)<\frac{\epsilon}{2^{n}}$ and

$$
F_{n}(x)+H_{n}(x)=(\mathcal{L}) \int_{a}^{x} \underline{D} F_{n}(t) d t+H_{n}(x)=(\mathcal{L}) \int_{a}^{x} u_{n}(t) d t .
$$

Clearly

$$
\begin{aligned}
F_{P_{n}}(x)+H_{n}(x) & =F_{n}(x)+H_{n}(x)+h_{n}(x) \\
& =(\mathcal{L}) \int_{a}^{x} u_{n}(t) d t+h_{n}(x) .
\end{aligned}
$$

Since $u_{n}$ is lower semicontinuous on $[a, b]$, from [13] (vol. II, p. 153) it follows that there exists a constant $\alpha_{n} \in \mathbb{R}$ such that $u_{n}(x) \geq \alpha_{n}$ for each $x \in[a, b]$. Let $a \leq x<y \leq b$. Then

$$
\left(F_{P_{n}}+H_{n}\right)(y)-\left(F_{P_{n}}+H_{n}\right)(x)=(\mathcal{L}) \int_{x}^{y} u_{n}(t) d t+h_{n}(y)-h_{n}(x) \geq \alpha_{n}(y-x),
$$


so $F_{P_{n}}+H_{n} \in \underline{L}$ on $[a, b]$. Let $H:[a, b] \rightarrow \mathbb{R}$ and $H(x)=\sum_{n=1}^{\infty} H_{n}(x)$. By Theorem 11 of [13] (p. 142) it follows that

$$
H(x)=(\mathcal{L}) \int_{a}^{x} \sum_{n=1}^{\infty}\left(u_{n}(t)-\underline{D} F_{n}(t)\right) d t .
$$

Clearly $H(a)=0, H(b)<\epsilon$ and $H$ is increasing and $A C$ on $[a, b]$; so we obtain (i) and (ii).

We have

$$
F(x)+H(x)=F(x)+H_{n}(x)+\sum_{\substack{i=1 \\ i \neq n}}^{\infty} H_{i}(x) \in \underline{L} \text { on } P_{n}
$$

(since $\sum_{\substack{i=1 \\ i \neq n}}^{\infty} H_{i}(x)$ is an increasing function on $\left.[a, b]\right)$. Hence we obtain (iii).

(iv) follows from (ii) and (iii).

Lemma 6. Let $F:[a, b] \rightarrow \mathbb{R}, F \in[V B G] \cap(N), F(a)=0$. Then there exist $M_{n}, m_{n}:[a, b] \rightarrow \mathbb{R}, n=1,2, \ldots$, having the following properties.

(i) $M_{n}(a)=m_{n}(a)=0$;

(ii) $M_{n}-F$ and $F-m_{n}$ are increasing on $[a, b]$;

(iii) $M_{n} \in[\underline{A C G}]$ and $m_{n} \in[\overline{A C G}]$ on $[a, b]$;

(iv) The sequences $\left\{\left(M_{n}-F\right)(b)\right\}_{n}$ and $\left\{\left(F-m_{n}\right)(b)\right\}_{n}$ are convergent to 0 .

Proof. Since $F \in[V B G] \cap(N)$ on $[a, b]$, there exists a sequence $\left\{P_{i}\right\}_{i}$ of closed sets such that $F \in V B \cap(N)$ on each $P_{i}$. We may suppose without loss of generality that each $P_{i}$ contains the points $a$ and $b$. Clearly $F_{P_{i}} \in V B \cap(N)$ on $[a, b]$. Fix a positive integer $i$. By Corollary 2, there exists a sequence $\left\{h_{i, n}\right\}_{n}, h_{i, n}:[a, b] \rightarrow \mathbb{R}$ having the following properties.

1) $h_{i, n}(a)=0$ for each $n$;

2) $h_{i, n}$ is increasing on $[a, b]$ for each $n$;

3) $F_{P_{i}}+h_{i, n} \in[\underline{A C G}]$ and $F_{P_{i}}-h_{i, n} \in[\overline{A C} G]$ on $[a, b]$ for each $n$;

4) $h_{i, n}(b)<\frac{1}{2^{i+n}}$.

Let $H_{n}:[a, b] \rightarrow \mathbb{R}, H_{n}=\sum_{i=1}^{\infty} h_{i, n}$. Then $H_{n}(a)=0, H_{n}$ is increasing on $[a, b]$ and

$$
H_{n}(b)<\sum_{i=1}^{\infty} \frac{1}{2^{i+n}}=\frac{1}{2^{n}}
$$


Let $M_{n}, m_{n}:[a, b] \rightarrow \mathbb{R}, M_{n}=F+H_{n}$ and $m_{n}=F-H_{n}$. Then (i), (ii) and (iv) follow by 1), 2) and (1).

(iii) We have

$$
M_{n}=F+h_{i, n}+\sum_{\substack{j=1 \\ j \neq i}}^{\infty} h_{j, n} .
$$

But $F+h_{i, n}=F_{P_{i}}+h_{i, n} \in[\underline{A C G}]$ on $P_{i}$ and $F-h_{i, n}=F_{P_{i}}-h_{i, n} \in[\overline{A C} G]$ on $P_{i}$ (see 3)). Since $\sum_{\substack{j=1 \\ j \neq i}}^{\infty} h_{j, n}$ defines an increasing bounded function on $[a, b]$, it follows that $M_{n} \in[\underline{A C G}]$ and $m_{n} \in[\overline{A C} G]$ on $[a, b]$.

Lemma 7. Let $F, F_{n}: P \rightarrow \mathbb{R}, n=1,2, \ldots, P \subset[a, b]$. If $F_{n}-F$ is increasing, $F_{n} \rightarrow F\left[\right.$ unif] on $P$ and $F_{n} \in \underline{A C}$ on $P$, then $F \in \underline{A C}$ on $P$.

Proof. Since $F_{n} \rightarrow F[$ unif] on $P$, for $\epsilon>0$, there exists a positive integer $n_{\epsilon}$ such that

$$
-\frac{\epsilon}{4} \leq F_{n}(x)-F(x)<\frac{\epsilon}{4}, \quad(\forall) n \geq n_{\epsilon} .
$$

Let $\delta_{\epsilon}>0$ be given for $\epsilon / 2$ by the fact that $F_{n_{\epsilon}} \in \underline{A C}$ on $P$. Let $\left\{\left[a_{j}, b_{j}\right]\right\}$, $j=1,2, \ldots, m$ be a finite set of nonoverlapping closed intervals with endpoints in $P$ such that $\sum_{j=1}^{m}\left(b_{j}-a_{j}\right)<\delta_{\epsilon}$. Since $F_{n_{\epsilon}}-F$ is increasing on $P$, by (2) we have

$$
\begin{gathered}
\sum_{j=1}^{m}\left(F\left(b_{j}\right)-F\left(a_{j}\right)\right)= \\
\left.=\sum_{j=1}^{m}\left(F-F_{n_{\epsilon}}\right)\left(b_{j}\right)-\left(F-F_{n_{\epsilon}}\right)\left(a_{j}\right)\right)+\sum_{j=1}^{m}\left(F_{n_{\epsilon}}\left(b_{j}\right)-F_{n_{\epsilon}}\left(a_{j}\right)\right)> \\
>-\frac{\epsilon}{2}-\frac{\epsilon}{2}=-\epsilon,
\end{gathered}
$$

hence $F \in \underline{A C}$ on $P$.

Lemma 8. Let $P \subseteq[a, b]$ be a Borel set, and let $F, M_{n}, m_{n}: P \rightarrow \mathbb{R}, n=$ $1,2, \ldots$, have the following properties.

- $M_{n}, m_{n} \in V B$ on $P$;

- $M_{n} \rightarrow F[u n i f]$ and $m_{n} \rightarrow F[u n i f]$ on $P$;

- $M_{n}-F$ and $F-m_{n}$ are bounded and increasing on $P$;

- $M_{n} \in \underline{M}$ and $m_{n} \in \bar{M}$ on $P$.

Then $F \in(N)$ on $P$. 
Proof. Since $F=\left(F-m_{n}\right)+m_{n}$, it follows that $F \in V B$ on $P$. Suppose on the contrary that $F \notin(N)$ on $P$. Then by Lemma 7, (iii) of [4], $F \notin \bar{M} \cap \underline{M}$ on $P$. Consider for example that $F \notin \underline{M}$ on $P$. From the definition of $\underline{M}$, it follows that there exists a compact set $Q$ such that $F \in V B \cap \mathcal{C}$ on $Q$, but $F \notin \underline{A C}$ on $Q$. We have $M_{n}=\left(M_{n}-F\right)+F \in \mathcal{C}_{i}$ on $Q$, because $M_{n}-F$ is increasing and $F$ is continuous on $Q$. Then $M_{n} \in V B \cap \mathcal{C}_{i} \cap \underline{M}$ on $Q$. Hence each $M_{n} \in \underline{A C}$ on $Q$ (see Theorem 3, (i), 1) and 4) of [4]). By Lemma 7, $F \in \underline{A C}$ on $Q$, a contradiction.

\section{The $\mathcal{L}$ DG Integral of C. M. Lee}

In this section we suppose that $u \mathcal{L} \subset\{F: \mathbb{R} \rightarrow \mathbb{R}\}$ is an upper semilinear space, contained in $u C M$ and satisfying property $(*)$ (see Definition $15)$. Clearly $\mathcal{L}=u \mathcal{L} \cap(-u \mathcal{L})$ is a linear space satisfying property $(*)$. Let $\mathcal{L}[a, b]=\left\{F:[a, b] \rightarrow \mathbb{R}: F_{a, b} \in \mathcal{L}\right\}$.

Definition 18. A function $f:[a, b] \rightarrow \overline{\mathbb{R}}$ is said to be $\mathcal{L} D G$-integrable on $[a, b]$ if there exists $F \in[A C G]$ on $[a, b]$ and $F \in \mathcal{L}([a, b])$, such that $F_{a p}^{\prime}(x)=f(x)$ a.e. on $[a, b]$. The function $F$ is said to be an indefinite $\mathcal{L} D G$-integral of $f$ on $[a, b]$ and we write $\mathcal{L} D G \int_{a}^{b} f(t) d t=F(b)-F(a)$.

Proposition 2. The $\mathcal{L} D G$ integral is well defined.

Proof. Let $F$ and $G$ be indefinite $\mathcal{L D} G$-integrals of $f$ on $[a, b]$. Then $F, G \in$ $\mathcal{L}[a, b], F, G \in[A C G]$ on $[a, b]$ and $F_{a p}^{\prime}=f=G_{a p}^{\prime}$ a.e. on $[a, b]$. It follows that $F-G \in \mathcal{L}[a, b], F-G \in[A C G]$ on $[a, b]$ and $(F-G)_{a p}^{\prime}=0$ a.e. on $[a, b]$. By Theorem A, a), $F-G$ is constant on $[a, b]$. Hence

$$
\mathcal{L} D G \int_{a}^{b} f(t) d t=F(b)-F(a)=G(b)-G(a) .
$$

\section{Remark 3.}

(i) If in Definition $18, \mathcal{L}=\mathcal{C}$, then we obtain the wide Denjoy integral.

(ii) If $\mathcal{L}=\mathcal{C}_{a p}$, then we obtain the $\beta$-Ridder integral [17] (that is also called the $A D$-integral of Kubota [8]). In fact Ridder gave three equivalent definitions for this integral: Definitions $2^{a}$ and Definition $2^{b}$ of [15] (p. 2), and Definition 7 of [17] (p. 148).

(iii) The fact that the $\beta$-Ridder integral is equivalent to the $A D$ integral is stated explicitly by Kubota in [10] (p. 219). 
Theorem 2. Let $f, g:[a, b] \rightarrow \overline{\mathbb{R}}$.

(i) If fand $g$ are $\mathcal{L} D G$ integrable on $[a, b]$, then for every $\alpha, \beta \in \mathbb{R}$ the function $\alpha f+\beta g$ is $\mathcal{L} D G$ integrable on $[a, b]$ and

$$
\mathcal{L} D G \int_{a}^{b}(\alpha f+\beta g)(t) d t=\alpha \cdot \mathcal{L} D G \int_{a}^{b} f(t) d t+\beta \cdot \mathcal{L} D G \int_{a}^{b} g(t) d t .
$$

(ii) If $f$ is $\mathcal{L} D G$-integrable on $[a, b]$ and $f=g$ a.e. on $[a, b]$, then $g$ is $\mathcal{L} D G$ integrable and $\mathcal{L} D G \int_{a}^{b} f(t) d t=\mathcal{L} D G \int_{a}^{b} g(t) d t$.

(iii) If $f$ is $\mathcal{L} D G$-integrable on $[a, b]$, then $f$ is $\mathcal{L} D G$-integrable on any subinterval $[\alpha, \beta]$ of $[a, b]$.

(iv) If $a<c<b$ and $f$ is $\mathcal{L} D G$ integrable on both, $[a, c]$ and $[c, b]$, then $f$ is $\mathcal{L} D G$ integrable on $[a, b]$ and

$$
\mathcal{L} D G \int_{a}^{c} f(t) d t+\mathcal{L} D G \int_{c}^{b} f(t) d t=\mathcal{L} D G \int_{a}^{b} f(t) d t .
$$

Proof. (i) and (ii) are obvious.

(iii) Since $f$ is $\mathcal{L} D G$ integrable on $[a, b]$, there exists $F \in[A C G]$ on $[a, b]$, $F \in \mathcal{L}([a, b])$, such that $F_{a p}^{\prime}=f$ a.e. on $[a, b]$. Since $\mathcal{L}$ satisfies the property $(*)$, we have that $F_{\mid[\alpha, \beta]} \in \mathcal{L}[\alpha, \beta]$ whenever $[\alpha, \beta] \subset[a, b]$. Clearly $F \in[A C G]$ on $[\alpha, \beta]$. Therefore $f$ is $\mathcal{L} D G$ integrable on $[\alpha, \beta]$ and

$$
\mathcal{L} D G \int_{\alpha}^{\beta} f(t) d t=F(\beta)-F(\alpha) .
$$

(iv) Let $F \in \mathcal{L}[a, c]$ such that $F$ is $[A C G]$ on $[a, c]$ and $F_{a p}^{\prime}=f$ a.e. on $[a, c]$. Let $G \in \mathcal{L}[c, b]$ such that $G$ is $[A C G]$ on $[c, b]$ and $G_{a p}^{\prime}=f$ a.e. on $[c, b]$. Let $H: \mathbb{R} \rightarrow \mathbb{R}, H=F_{a, c}+G_{c, b}$. Then $H=H_{a, b} \in \mathcal{L}$. It follows that $H_{\mid[a, b]} \in \mathcal{L}[a, b]$. Clearly $H \in[A C G]$ on $[a, b], H_{a p}^{\prime}=f$ a.e. on $[a, b]$ and

$$
\begin{gathered}
\mathcal{L} D G \int_{a}^{b} f(t) d t=H(b)-H(a)=F(c)-F(a)+G(b)-G(c)= \\
=\mathcal{L} D G \int_{a}^{c} f(t) d t+\mathcal{L} D G \int_{c}^{b} f(t) d t .
\end{gathered}
$$




\section{$7 \quad$ Sarkhel Type Integrals}

In this section we suppose that $u \mathcal{L} \subset\{F: \mathbb{R} \rightarrow \mathbb{R}\}$ is an upper semilinear space, contained in lower internal and satisfying property $(*)$ (see Definition $15)$. Clearly $\mathcal{L}=u \mathcal{L} \cap(-u \mathcal{L})$ is a linear space satisfying property $(*)$. Let $\mathcal{L}[a, b]=\left\{F:[a, b] \rightarrow \mathbb{R}: F_{a, b} \in \mathcal{L}\right\}$.

Definition 19. A function $f:[a, b] \rightarrow \overline{\mathbb{R}}$ is said to be $\mathcal{L} S$ integrable on $[a, b]$ if there exists a function $F \in \mathcal{L}[a, b], F \in[V B G] \cap(N)$ on $[a, b]$ such that $F_{a p}^{\prime}(x)=f(x)$ a.e. on $[a, b]$. The function $F$ is said to be an indefinite $\mathcal{L} S$ integral of $f$ on $[a, b]$ and we write $\mathcal{L} S \int_{a}^{b} f(t) d t=F(b)-F(a)$.

Proposition 3. The $\mathcal{L} S$ integral is well defined.

Proof. Let $F$ and $G$ be indefinite $\mathcal{L} S$ integrals of $f$ on $[a, b]$. Then $F, G \in$ $\mathcal{L}[a, b], F, G \in(N) \cap[V B G]$ on $[a, b]$ and $F_{a p}^{\prime}=f=G_{a p}^{\prime}$ a.e. on $[a, b]$. It follows that $F-G \in \mathcal{L}[a, b], F-G \in(N) \cap[V B G]$ on $[a, b]$ (because $(N) \cap[V B G]$ is a linear space (see Theorem A, g)) and $(F-G)_{a p}^{\prime}=0$ a.e. on $[a, b]$. By Theorem A, b), $F-G$ is constant on $[a, b]$. Hence $\mathcal{L} S \int_{a}^{b} f(t) d t=F(b)-F(a)=$ $G(b)-G(a)$.

\section{Remark 4.}

(i) A result similar to Theorem 2 is also true for the $\mathcal{L} S$ integral.

(ii) In Definition 19, the condition $(N)$ may be replaced by the condition $(M)$ (see Theorem A, g)).

(iii) If in Definition $19, \mathcal{L}=\mathcal{C}$, then we obtain the wide Denjoy integral, because $[V B G] \cap(N) \cap \mathcal{C}=[A C G] \cap \mathcal{C}=A C G \cap \mathcal{C}$ (see Theorem 6.8 of [19], p. 228).

(iv) If in Definition $19, \mathcal{L}=\mathcal{C}_{a p}$, then we obtain an integral more general than the $\beta$-Ridder integral. This follows from an example of Sarkhel and Kar (see Example 3.1 and Theorem 3.6 of [23]), who constructed a function $F:[a, b] \rightarrow \mathbb{R}$ with the following properties.

- $F \in \mathcal{C}_{a p}$ on $[a, b]$.

- $F \in[V B G] \cap(N)$ on $[a, b]$.

- $F$ is neither $\underline{A C G}$ nor $\overline{A C} G$ on $[a, b]$. Hence $F \notin A C G$ on $[a, b]$.

(v) The idea of using $[V B G] \cap(N)$ in the definition of an integral comes from Sarkhel, who used the condition $P A C$ in defining his $T D$ integral [21] and $T P$ integral [20]. But $P A C=[V B G] \cap(N)$ on a compact set (see Theorem 3.6 of [23]). Sarkhel and Kar showed that $P A C$ is a linear space on a compact set (see Corollary 3.1.1 of [23]). 


\section{Generalized Sarkhel Type Integrals}

In this section we suppose that $u \mathcal{L} \subset\{F: \mathbb{R} \rightarrow \mathbb{R}\}$ is an upper semilinear space, contained in lower internal and satisfying property $(*)$ (see Definition $15)$. Clearly $\mathcal{L}=u \mathcal{L} \cap(-u \mathcal{L})$ is a linear space satisfying property $(*)$. Let $\mathcal{L}[a, b]=\left\{F:[a, b] \rightarrow \mathbb{R}: F_{a, b} \in \mathcal{L}\right\}$.

Definition 20. A function $f:[a, b] \rightarrow \overline{\mathbb{R}}$ is said to be $\mathcal{L} S G$ integrable on $[a, b]$ if there exists a function $F \in \mathcal{L}[a, b], F \in \mathcal{B}_{1} \cap V B G \cap(N)$ on $[a, b]$ such that $F_{a p}^{\prime}(x)=f(x)$ a.e. on $[a, b]$. The function $F$ is said to be an indefinite $\mathcal{L} S G$ integral of $f$ on $[a, b]$ and we write $\mathcal{L} S G \int_{a}^{b} f(t) d t=F(b)-F(a)$.

Proposition 4. The $\mathcal{L} S G$ integral is well defined.

Proof. Let $F$ and $G$ be indefinite $\mathcal{L} S G$ integrals of $f$ on $[a, b]$. Then $F, G \in$ $\mathcal{L}[a, b], F, G \in(N) \cap V B G \cap \mathcal{B}_{1}$ on $[a, b]$ and $F_{a p}^{\prime}=f=G_{a p}^{\prime}$ a.e. on $[a, b]$. It follows that $F-G \in \mathcal{L}[a, b], F-G \in(N) \cap V B G \cap \mathcal{B}_{1}$ on $[a, b]$ (because $(N) \cap V B G \cap \mathcal{B}_{1}$ is a linear space, see Theorem A, f)) and $(F-G)_{a p}^{\prime}=0$ a.e. on $[a, b]$. By Theorem A, b), $F-G$ is constant on $[a, b]$. Hence $\mathcal{L} S G \int_{a}^{b} f(t) d t=$ $F(b)-F(a)=G(b)-G(a)$.

\section{Remark 5.}

(i) A result similar to Theorem 2 is also true for the $\mathcal{L} S G$ integral.

(ii) In Definition 20, the condition $(N)$ may be replaced by Foran's condition $(M)$ (see Theorem A, f)).

(iii) If in Definition $20, \mathcal{L}=\mathcal{C}$, then we obtain the wide Denjoy integral, because $V B G \cap(N) \cap \mathcal{C}=A C G \cap \mathcal{C}$ (see Theorem 6.8 of [19], p. 228).

(iv) If in Definition 20, $\mathcal{L}=\mathcal{C}_{a p}$, then we obtain Gordon's Definition 3 of [7]. But in proving the uniqueness of his integral, he neglected to show that the difference of two $V B G \cap \mathcal{C}_{a p} \cap(N)$ functions satisfies $(N)$ on $[a, b]$.

(v) Question. If $\mathcal{L}=\mathcal{C}_{a p}$, then is the $\mathcal{L} S G$ integral a strict generalization of the $\mathcal{L} S$ integral?

(vi) For a suitable choice of the class $\mathcal{L}$, the $\mathcal{L} S G$ integral contains the integrals studied by Sarkhel, De and Kar in [22], [20], [21], [23].

\section{Perron-Ridder-Lee Type Integrals}

Definition 21. Let $u \mathcal{L} \subset\{F: \mathbb{R} \rightarrow \mathbb{R}\}$ be an upper semilinear space, closed under uniform convergence. 
- $\mathcal{L}=u \mathcal{L} \cap(-u \mathcal{L})$.

- Let $u \mathcal{L}[a, b]=\left\{F:[a, b] \rightarrow \mathbb{R}: F_{a, b} \in u \mathcal{L}\right\}$.

- $\mathcal{L}[a, b]=\left\{F:[a, b] \rightarrow \mathbb{R}: F_{a, b} \in \mathcal{L}\right\}$.

Let $f:[a, b] \rightarrow \overline{\mathbb{R}}$.

- Suppose that $\mathcal{A}$ is an upper semilinear space satisfying the condition $(*)$, such that $\mathcal{C} \subseteq \mathcal{L} \subseteq \mathcal{A} \subseteq u \mathcal{L} \subset u C M$. Let $\mathcal{A}[a, b]=\{F:[a, b] \rightarrow \mathbb{R}$ : $\left.F_{a, b} \in \mathcal{A}\right\}$. We define the following classes of majorants.

$-\mathcal{A} \overline{\mathcal{M}}_{0}(f ;[a, b])=\{M:[a, b] \rightarrow \mathbb{R}: M(a)=0 ; M \in \mathcal{A}([a, b]) ;$ $M \in[(\underline{L} \cap A C) G]$ on $[a, b] ; M_{a p}^{\prime}(x) \geq f(x)$ a.e. on $\left.[a, b]\right\}$.

$-\mathcal{A} \overline{\mathcal{M}}_{1}(f ;[a, b])=\{M:[a, b] \rightarrow \mathbb{R}: M(a)=0 ; M \in \mathcal{A}([a, b]) ;$ $M \in[\underline{L} G]$ on $[a, b] ; M_{a p}^{\prime}(x) \geq f(x)$ a.e. on $\left.[a, b]\right\}$.

$-\mathcal{A} \overline{\mathcal{M}}_{2}(f ;[a, b])=\{M:[a, b] \rightarrow \mathbb{R}: M(a)=0 ; M \in \mathcal{A}([a, b]) ;$ $M \in[\underline{A C G}]$ on $[a, b] ; M_{a p}^{\prime}(x) \geq f(x)$ a.e. on $\left.[a, b]\right\}$.

- Suppose that $\mathcal{A}$ is an upper semilinear space satisfying the condition $(*)$, such that $\mathcal{C} \subseteq \mathcal{L} \subset \mathcal{A} \subseteq u \mathcal{L} \subset$ lower internal. Let $\mathcal{A}[a, b]=\{F:[a, b] \rightarrow$ $\left.\mathbb{R}: F_{a, b} \in \mathcal{A}\right\}$. We define the following classes of majorants.

$-\mathcal{A} \overline{\mathcal{M}}_{3}(f ;[a, b])=\{M:[a, b] \rightarrow \mathbb{R}: M(a)=0 ; M \in \mathcal{A}([a, b]) ;$ $M \in[V B G] \cap \underline{M}$ on $[a, b] ; M_{a p}^{\prime}(x) \geq f(x)$ a.e. on $\left.[a, b]\right\}$.

$-\mathcal{A} \overline{\mathcal{M}}_{4}(f ;[a, b])=\{M:[a, b] \rightarrow \mathbb{R}: M(a)=0 ; M \in \mathcal{A}([a, b]) ;$ $M \in V B G \cap \underline{M} \cap \mathcal{B}_{1}$ on $[a, b] ; M_{a p}^{\prime}(x) \geq f(x)$ a.e. on $\left.[a, b]\right\}$.

We define the following classes of minorants.

- $\mathcal{A} \underline{\mathcal{M}}_{j}(f ;[a, b])=\left\{m:[a, b] \rightarrow \mathbb{R}:-m \in \mathcal{A} \overline{\mathcal{M}}_{j}(-f ;[a, b])\right\}, j=$ $0,1,2,3,4$.

For each $j=0,1,2,3,4$ we define the following integral.

- If $\mathcal{A} \overline{\mathcal{M}}_{j}(f ;[a, b]) \neq \emptyset$, then we denote by $\mathcal{A} \bar{I}_{j}(f ;[a, b])$ the lower bound of all $M(b), M \in \mathcal{A} \overline{\mathcal{M}}_{j}(f ;[a, b])$.

- If $\mathcal{A} \underline{\mathcal{M}}_{j}(f ;[a, b]) \neq \emptyset$, then we denote by $\mathcal{A} \underline{I}_{j}(f ;[a, b])$ the upper bound of all $m(b), m \in \mathcal{A M}_{j}(f ;[a, b])$.

- $f$ is said to have an $\mathcal{A P}_{j}$ integral on $[a, b]$ if

$$
\mathcal{A} \overline{\mathcal{M}}_{j}(f ;[a, b]) \times \mathcal{A} \underline{\mathcal{M}}_{j}(f ;[a, b]) \neq \emptyset
$$

and

$$
\mathcal{A} \bar{I}_{j}(f ;[a, b])=\mathcal{A} \underline{I}_{j}(f ;[a, b])=\mathcal{A} \mathcal{P}_{j} \int_{a}^{b} f(t) d t
$$


Remark 6. (i) In the definition of $\mathcal{A} \overline{\mathcal{M}}_{3}(f ;[a, b])$, the condition $\underline{M}$ may be replaced by $(\underline{N})$ (see Theorem A, e)).

(ii) In the definition of $\mathcal{A} \overline{\mathcal{M}}_{4}(f ;[a, b])$, the condition $\underline{M}$ may be replaced by $(\underline{N})$ (see Theorem A, d)).

(iii) In the definition of $\mathcal{A} \overline{\mathcal{M}}_{2}(f ;[a, b])$ the condition $[\underline{A C G}]$ may be replaced

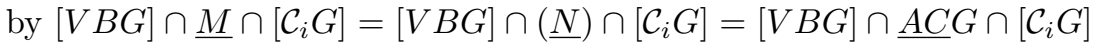
(see Theorem A, d) and Corollary 2.21.1, (iii) of [2]).

(iv) If $\mathcal{A}=u \mathcal{L}$, then $\mathcal{A P}_{2}$ is in fact the $\mathcal{L} P G$ integral of C. M. Lee [11].

(v) For $\mathcal{A}=\mathcal{C}_{a p}$, the $\mathcal{A} \overline{\mathcal{M}}_{2}(f ;[a, b])$ majorants are exactly as in the following definitions of Ridder: Definition A of [15] (p. 3), Definition 6 of [16] (p. 12), Definition $C_{1}$ of [17] (p. 148); Definition $C_{1}$ of [18] (p. 176). Also the $\mathcal{A M}_{2}(f ;[a, b])$ minorants are exactly as in Definition B of [15] (p. 3) and Definition $D_{1}$ of [17] (p. 149). In the same conditions the $\mathcal{A P}_{2}$ integral is exactly as in Ridder's Definition 3 of [15] (p. 5) and Definition 8 of [17] (p. 149). At p. 6 of [15] Ridder asserts that this integral is equivalent with his $\beta$-integral (Definitions $2^{a}$ and $2^{b}$ of [15]; see also Remark 3). His proof is based on the following fact. if $\left(M_{k}, m_{k}\right) \in$ $\mathcal{A} \overline{\mathcal{M}}_{2}(f ;[a, b]) \times \mathcal{A} \mathcal{M}_{2}(f ;[a, b])$, then there exists a sequence $\left\{E_{j}\right\}_{j}$ of perfect sets and a countable set $H$ (possibly empty), with $[a, b]=H \cup$ $\left(\cup_{j=1}^{\infty} E_{j}\right)$, such that each $M_{k} \in \underline{A C}$ and each $m_{k} \in \overline{A C}$ on each $E_{j}$. This is true, but it needs proof (that is not easy).

(vi) For $\mathcal{A}=\mathcal{C}_{a p}$ the $\mathcal{A P}_{2}(f ;[a, b])$ integral is exactly as in Kubota's Definition 8 of [9] (p. 740), and he calls this integral $A P^{*}$ integral. Although Kubota doesn't mention Ridder's papers [15], [17], [18], [16], he also proves the equivalence between the $A P^{*}$ integral and the $A D$-integral $=\beta$-Ridder integral (see Theorem 3.6 of [9]), but he makes the same omission as Ridder did (see (v)).

(vii) For $\mathcal{A}=\mathcal{C}$, the $\mathcal{A} \overline{\mathcal{M}}_{2}(f ;[a, b])$ majorants are exactly as in Ridder's Definition 13 of [16] (p. 15).

Theorem 3. Let $f:[a, b] \rightarrow \overline{\mathbb{R}}$.

(i) $\mathcal{A} \overline{\mathcal{M}}_{0}(f ;[a, b]) \subseteq \mathcal{A} \overline{\mathcal{M}}_{1}(f ;[a, b]) \subseteq \mathcal{A} \overline{\mathcal{M}}_{2}(f ;[a, b])$.

(ii) $\mathcal{A} \overline{\mathcal{M}}_{3}(f ;[a, b]) \subseteq \mathcal{A} \overline{\mathcal{M}}_{4}(f ;[a, b])$.

(iii) If $\mathcal{A} \subset$ lower internal, then $\mathcal{A} \overline{\mathcal{M}}_{2}(f ;[a, b]) \subseteq \mathcal{A} \overline{\mathcal{M}}_{3}(f ;[a, b])$.

Moreover, for each $j=0,1,2,3,4$, if

$$
(M, m) \in \mathcal{A} \overline{\mathcal{M}}_{j}(f ;[a, b]) \times \mathcal{A \mathcal { M }}_{j}(f ;[a, b]),
$$


then $M-m$ is increasing on $[a, b]$. Hence $M(b) \geq m(b)$. This implies

$$
M(b) \geq \mathcal{A} \bar{I}_{j}(f ;[a, b]) \geq \mathcal{A} \underline{I}_{j}(f ;[a, b]) \geq m(b) .
$$

Proof. The proof of (i) follows by the fact that we always have $\underline{L} \subseteq \underline{A C}$ on a set, and the proof of (ii) is obvious. (iii) follows because $\underline{A C} \subset V B$ on a closed set (see Theorem 2.11.1, (vi) of [2]).

That $M-m$ is increasing on $[a, b]$ for $j=0,1,2$ follows by Theorem A, a), and for $j=3,4$ see Theorem A, c). The relation (3) follows by definitions.

Lemma 9. The function $f:[a, b] \rightarrow \overline{\mathbb{R}}$ is $\mathcal{A P}_{j}$ integrable on $[a, b]$ for $j=$ $0,1,2,3,4$, if and only if for every $\epsilon>0$ there exists

$$
(M, m) \in \mathcal{A} \overline{\mathcal{M}}_{j}(f ;[a, b]) \times \mathcal{A} \underline{\mathcal{M}}_{j}(f ;[a, b]) \neq \emptyset
$$

such that $M(b)-m(b)<\epsilon$.

Proof. The proof follows by Theorem 3 .

Corollary 3. Let $f:[a, b] \rightarrow \overline{\mathbb{R}}$.

(i) If $f$ is $\mathcal{A P}_{j}$ integrable on $[a, b], j=0,1$, then $f$ is $\mathcal{A P}_{j+1}$ integrable on $[a, b]$ and $\mathcal{A P}_{j} \int_{a}^{b} f(t) d t=\mathcal{A P}_{j+1} \int_{a}^{b} f(t) d t$. Moreover, if $f$ is $\mathcal{A P}_{2}$ integrable on $[a, b]$, then $f$ is $\mathcal{A P}_{1}$ integrable on $[a, b]$. Hence the two integrals are equivalent.

(ii) If $\mathcal{A} \subset$ lower internal and $f$ is $\mathcal{A P}_{2}$ integrable on $[a, b]$, then $f$ is $\mathcal{A P}_{3}$ integrable on $[a, b]$ and $\mathcal{A P}_{2} \int_{a}^{b} f(t) d t=\mathcal{A P}_{3} \int_{a}^{b} f(t) d t$.

(iii) If $f$ is $\mathcal{A P}_{3}$ integrable on $[a, b]$, then $f$ is $\mathcal{A P}_{4}$ integrable on $[a, b]$ and $\mathcal{A P}_{3} \int_{a}^{b} f(t) d t=\mathcal{A P}_{4} \int_{a}^{b} f(t) d t$.

Proof. We prove for example (i). By Theorem 3, (i) we have

$$
\mathcal{A} \bar{I}_{1}(f ;[a, b]) \geq \mathcal{A} \bar{I}_{2}(f ;[a, b]) \geq \mathcal{A} \underline{I}_{2}(f ;[a, b]) \geq \mathcal{A} \underline{I}_{1}(f ;[a, b]) .
$$

Suppose that $f$ is $\mathcal{A} \mathcal{P}_{2}$ integrable on $[a, b]$. Then $\mathcal{A} \bar{I}_{2}(f ;[a, b])=\mathcal{A} \underline{I}_{2}(f ;[a, b])$ $\in \mathbb{R}$. For $\epsilon>0$, let $M \in \mathcal{A} \overline{\mathcal{M}}_{2}(f ;[a, b])$ such that $M(b)<\mathcal{A} \bar{I}_{2}(f ;[a, b])+\frac{\epsilon}{2}$. Then $M \in \mathcal{A}[a, b], M \in[\underline{A C G}]$ on $[a, b]$ and $M_{a p}^{\prime} \geq f$ a.e. on $[a, b]$. By Lemma 5 , there exists $H:[a, b] \rightarrow \mathbb{R}$ such that $H(a)=0, H(b)<\epsilon / 2, H$ is increasing and $A C$ on $[a, b]$ and $U:=M+H \in[\underline{L} G]$ on $[a, b]$. Since $\mathcal{C} \subset \mathcal{A}$ and $\mathcal{A}$ is an upper semilinear space, it follows that $U \in \mathcal{A}[a, b]$. We obtain that $U \in \mathcal{A} \overline{\mathcal{M}}_{1}(f ;[a, b])$ and $U(b) \leq \mathcal{A} \bar{I}_{2}(f ;[a, b])+\epsilon$. Hence

$$
\mathcal{A} \bar{I}_{1}(f ;[a, b]) \leq \mathcal{A P}_{2} \int_{a}^{b} f(t) d t .
$$


Similarly we obtain that

$$
\mathcal{A} \underline{I}_{1}(f ;[a, b]) \geq \mathcal{A} \mathcal{P}_{2} \int_{a}^{b} f(t) d t .
$$

Since we always have that $\mathcal{A} \underline{I}_{1}(f ;[a, b]) \leq \mathcal{A} \bar{I}_{1}(f ;[a, b])$,

$$
\mathcal{A P}_{1} \int_{a}^{b} f(t) d t=\mathcal{A P}_{2} \int_{a}^{b} f(t) d t
$$

Definition 22. A function $f: Q \rightarrow \overline{\mathbb{R}}$ is said to be $\mathcal{A} \mathcal{P}_{j}$ integrable on a bounded set $E \subset Q, j=0,1,2,3,4, a=\inf (E), b=\sup (E)$, if the function

$$
\tilde{f}_{E}:[a, b] \rightarrow \mathbb{R} \quad \tilde{f}_{E}(x)= \begin{cases}f(x) & \text { if } x \in E \\ 0 & \text { if } x \in[a, b] \backslash E\end{cases}
$$

is $\mathcal{A} \mathcal{P}_{j}$ integrable on $[a, b]$. We shall write

$$
\mathcal{A} \mathcal{P}_{j} \int_{E} f(t) d t=\mathcal{A} \mathcal{P}_{j} \int_{a}^{b} \tilde{f}_{E}(t) d t
$$

Clearly, for $E=[c, d]$ we have

$$
\mathcal{A} \mathcal{P}_{j} \int_{[c, d]} f(t) d t=\mathcal{A} \mathcal{P}_{j} \int_{c}^{d} f(t) d t .
$$

Theorem 4. If $f$ is $\mathcal{A} \mathcal{P}_{j}$ integrable on $[a, b], j=0,1,2,3,4$, and $[\alpha, \beta] \subset[a, b]$, then $f$ is $\mathcal{A P}_{j}$ integrable on $[\alpha, \beta]$. Moreover, if $a<\alpha<\beta$, then

$$
\mathcal{A P}_{j} \int_{a}^{\alpha} f(t) d t+\mathcal{A} \mathcal{P}_{j} \int_{\alpha}^{\beta} f(t) d t=\mathcal{A} \mathcal{P}_{j} \int_{a}^{\beta} f(t) d t
$$

Proof. By Theorem 3 and Lemma 9 it follows that for $\epsilon>0$ there exists

$$
(M, m) \in \mathcal{A} \overline{\mathcal{M}}_{j}(f ;[a, b]) \times \mathcal{A \mathcal { M }}_{j}(f ;[a, b]) \neq \emptyset
$$

such that $M-m$ is increasing on $[a, b]$ and $0 \leq M(b)-m(b)<\epsilon$. Let $M_{1}, m_{1}:[\alpha, \beta] \rightarrow \mathbb{R}$ by

$$
M_{1}(x)=M(x)-M(\alpha) \quad \text { and } \quad m_{1}(x)=m(x)-m(\alpha) .
$$

Since $\mathcal{A}$ satisfies property $(*)$,

$$
\left(M_{1}, m_{1}\right) \in \mathcal{A} \overline{\mathcal{M}}_{j}(f ;[\alpha, \beta]) \times \mathcal{A} \underline{\mathcal{M}}_{j}(f ;[\alpha, \beta])
$$


and $M_{1}(\beta)-m_{1}(\beta)<\epsilon$. Therefore by Lemma $9, f$ is $\mathcal{A P}_{j}$-integrable on the interval $[\alpha, \beta]$. We have

$$
\begin{gathered}
m(\alpha) \leq \mathcal{A} \mathcal{P}_{j} \int_{a}^{\alpha} f(t) d t \leq M(\alpha) \\
m_{1}(\beta)=m(\beta)-m(\alpha) \leq \mathcal{A} \mathcal{P}_{j} \int_{\alpha}^{\beta} f(t) d t \leq M(\beta)-M(\alpha)=M_{1}(\beta) \\
m(\beta) \leq \mathcal{A P}_{j} \int_{a}^{\beta} f(t) d t \leq M(\beta) .
\end{gathered}
$$

Therefore

$$
\begin{aligned}
& \mid \mathcal{A P}_{j} \int_{a}^{\beta} f(t) d t-\left(\mathcal{A P}_{j} \int_{a}^{\alpha} f(t) d t+\mathcal{A P}_{j} \int_{\alpha}^{\beta} f(t) d t\right) \mid \leq \\
& \leq M(\beta)-m(\beta)<\epsilon .
\end{aligned}
$$

Since $\epsilon$ is arbitrary, we obtain (4).

Definition 23. Let $f$ be an $\mathcal{A P}_{j}$ integrable function on $[a, b], j=0,1,2,3,4$. Then we define the indefinite $\mathcal{A P}_{j}$ integral of $f$ on $[a, b]$ by $F:[a, b] \rightarrow \mathbb{R}$, $F(a)=0$ and

$$
F(x)=\mathcal{A P}_{j} \int_{a}^{x} f(t) d t, \quad x \in(a, b] .
$$

Lemma 10. Let $F, M, m:[a, b] \rightarrow \mathbb{R}$ be functions such that

- $M$ and $-m$ are $\mathcal{C}_{i}$ on $[a, b]$;

- $M-F$ and $F-m$ are continuous on $[a, b]$.

Then $F$ is continuous on $[a, b]$.

Proof. $-F=-m+(m-F) \in \mathcal{C}_{i}$ on $[a, b]$ and $F=(F-M)+M \in \mathcal{C}_{i}$ on $[a, b]$. It follows that $F$ is continuous on $[a, b]$ (see Proposition 1).

Theorem 5. Let $f$ be an $\mathcal{A P}_{j}$-integrable function on $[a, b], j=0,1,2,3,4$, and $F(x)=\mathcal{A P}_{j} \int_{a}^{x} f(t) d t$. Suppose that

$$
(M, m) \in \mathcal{A} \overline{\mathcal{M}}_{j}(f ;[a, b]) \times \mathcal{A} \underline{\mathcal{M}}_{j}(f ;[a, b]) \neq \emptyset .
$$

Then

(i) $M-F$ and $F-m$ are increasing on $[a, b]$;

(ii) $F \in \mathcal{L}[a, b]$; 
(iii) $F$ is approximately derivable a.e. on $[a, b]$ and $F_{a p}^{\prime}(x)=f(x)$ a.e. on $[a, b]$.

Proof. (i) Let $a \leq x_{1}<x_{2} \leq b$. Let $M_{1}:\left[x_{1}, x_{2}\right] \rightarrow \mathbb{R}, M_{1}(x)=M(x)-$ $M\left(x_{1}\right)$. Then $M_{1} \in \mathcal{A} \underline{\mathcal{M}}_{j}\left(f ;\left[x_{1}, x_{2}\right]\right)$ (because $\mathcal{A}$ satisfies the property $\left.(*)\right)$; so

$$
M_{1}\left(x_{2}\right) \geq \mathcal{A} \mathcal{P}_{j} \int_{x_{1}}^{x_{2}} f(t) d t
$$

By Theorem 4, we have $M\left(x_{2}\right)-M\left(x_{1}\right) \geq F\left(x_{2}\right)-F\left(x_{1}\right)$.

(ii) For each positive integer $n$ there exists $M_{n} \in \mathcal{A} \underline{\mathcal{M}}_{j}(f ;[a, b])$ such that

$$
0 \leq M_{n}(x)-F(x)<\frac{1}{n}, \quad(\forall) x \in[a, b] .
$$

It follows that $\left\{M_{n}\right\}_{n}$ converges uniformly to $F$ on $[a, b]$. Hence $F \in u \mathcal{L}([a, b])$. Similarly $-F \in u \mathcal{L}([a, b])$. Hence $F \in \mathcal{L}[a, b]$.

(iii) Let $M_{0} \in \mathcal{A} \mathcal{M}_{j}(f ;[a, b])$. Then $M_{0} \in V B G \cap \mathcal{B}_{1}$ on $[a, b]$ and by (i), $M_{0}-F$ is increasing on $[a, b]$. It follows that $F=\left(F-M_{0}\right)+M_{0}$ is $V B G \cap \mathcal{B}_{1}$ on $[a, b]$. Hence $F$ is approximately derivable a.e. on $[a, b]$ (see Theorem 4.3 of [19], p. 222).

We show that $F_{a p}^{\prime}(x)=f(x)$ a.e. on $[a, b]$. For $\epsilon>0$ let

$$
(M, m) \in \mathcal{A} \underline{\mathcal{M}}_{j}(f ;[a, b]) \times \mathcal{A} \overline{\mathcal{M}}_{j}(f ;[a, b]) .
$$

such that $M(b)-m(b)<\epsilon^{2}$. Since $M, m \in V B G \cap \mathcal{B}_{1}$ on $[a, b]$, it follows that $M$ and $m$ are approximately derivable a.e. on $[a, b]$ and $m_{a p}^{\prime}(x) \leq f(x) \leq$ $M_{a p}^{\prime}(x)$ a.e. on $[a, b]$. So $f$ is finite a.e. on $[a, b]$. Let

$$
E=\left\{x \in[a, b]: f(x), F_{a p}^{\prime}(x), M_{a p}^{\prime}(x), m_{a p}^{\prime}(x) \text { are finite }\right\} .
$$

Then $E$ is a measurable set and $m(E)=b-a$. Let

$$
A_{\epsilon}=\left\{x \in E:\left|F_{a p}^{\prime}(x)-f(x)\right|>\epsilon\right\}
$$

and

$$
B_{\epsilon}=\left\{x \in E: M_{a p}^{\prime}(x)-m_{a p}^{\prime}(x)>\epsilon\right\} .
$$

Since $M-F$ and $F-m$ are increasing on $[a, b]$,

$$
M_{a p}^{\prime}(x) \geq F_{a p}^{\prime}(x) \geq m_{a p}^{\prime}(x), \quad(\forall) x \in E .
$$

Then $B_{\epsilon}$ is measurable and $A_{\epsilon} \subset B_{\epsilon}$. Since $M-m$ is increasing on $[a, b]$,

$$
\epsilon \cdot\left|B_{\epsilon}\right| \leq(\mathcal{L}) \int_{B_{\epsilon}}(M-m)^{\prime}(t) d t \leq
$$




$$
\leq(\mathcal{L}) \int_{a}^{b}(M-m)^{\prime}(t) d t \leq M(b)-m(b)<\epsilon^{2}
$$

(see Theorem 5 of [13], vol. I, p. 212). Hence $m\left(B_{\epsilon}\right)<\epsilon$; so $m\left(A_{\epsilon}\right)<\epsilon$. Let

$$
A=\left\{x \in E:\left|F_{a p}^{\prime}(x)-f(x)\right|>0\right\} .
$$

Then $A=\cup_{n=1}^{\infty} A_{\epsilon / 2^{n}}$. Hence $m(A)<\epsilon$. Since $\epsilon$ was arbitrary, it follows that $m(A)<0$. Hence $F_{a p}^{\prime}(x)=f(x)$ a.e. on $[a, b]$.

Theorem 6. Suppose that $\mathcal{A}$ is a class of the first type and let $f$ be an $\mathcal{A P}_{2}$ integrable function on $[a, b]$ and $F(x)=\mathcal{A P}_{2} \int_{a}^{x} f(t) d t$. Suppose that $(M, m) \in$ $\mathcal{A} \overline{\mathcal{M}}_{2}(f ;[a, b]) \times \mathcal{A} \underline{\mathcal{M}}_{2}(f ;[a, b]) \neq \emptyset$. Then

(i) $M-F$ and $F-m$ are increasing and continuous on $[a, b]$;

(ii) $F$ is $[\mathcal{C} G]$ on $[a, b]$;

(iii) $F \in[A C G]$ on $[a, b]$.

Proof. (i) Let $F(x)=\mathcal{A P}_{2} \int_{a}^{x} f(t) d t$. By Theorem 5, (i), (ii) it follows that $M-F$ and $F-m$ are increasing on $[a, b]$ and $F \in \mathcal{L}[a, b]$. Now the proof follows by the fact that $\mathcal{A}$ is a class of the first type.

(ii) Let $(M, m) \in \mathcal{A} \overline{\mathcal{M}}_{2}(f ;[a, b]) \times \mathcal{A} \underline{\mathcal{M}}_{2}(f ;[a, b])$. Then there exists $\left\{E_{i}\right\}_{i}$ a sequence of closed sets such that $[a, b]=\cup_{i=1}^{\infty} E_{i}$ and $M,-m \in \underline{A C} \subset V B$ on each $E_{i}$ (see for example Theorem 2.11.1, (vii) of [2]). Let

$$
U_{i}(x)= \begin{cases}M(x) & \text { if } x \in E_{i} \\ \text { linear } & \text { on the closure of each interval contiguous to } E_{i} \cup\{a, b\} .\end{cases}
$$

and

$$
L_{i}(x)= \begin{cases}m(x) & \text { if } x \in E_{i} \\ \text { linear } & \text { on the closure of each interval contiguous to } E_{i} \cup\{a, b\} .\end{cases}
$$

Then $U_{i}$ and $L_{i}$ are $\underline{A C}$ on $[a, b]$ (see Theorem 2.11.1, (xvii) of [2]). Let

$$
F_{i}(x)= \begin{cases}F(x) & \text { if } x \in E_{i} \\ \text { linear } & \text { on the closure of each interval contiguous to } E_{i} \cup\{a, b\} .\end{cases}
$$

But $U_{i}-F_{i}$ and $F_{i}-L_{i}$ are continuous on $[a, b]$ (see (i)). Also $U_{i},-L_{i} \in$ $\underline{A C} \subset \mathcal{C}_{i}$ (see Theorem 2.11.1, (xxi) of [2]). By Lemma 10, it follows that 
$F_{i}$ is continuous on $[a, b]$. Hence $F$ is continuous on each $E_{i}$. It follows that $F \in[\mathcal{C} G]$ on $[a, b]$.

(iii) Let $U:[a, b] \rightarrow \mathbb{R}$ be a major function of $f$ on $[a, b]$ and let $E_{i}$ be defined as in the proof of (ii). Then $U=F+(U-F)$ is continuous and $V B$ on $E_{i}$. But $U \in \underline{A C G} \subset(\underline{N}) \subset \underline{M}$ (see Theorem 2.20.1, Theorem 2.32.2, (i), (iv) and Theorem 2.23 .1 of [2]). Hence $U \in \underline{A C}$ on $E_{i}$. Let $\left\{M_{n}\right\}_{n} \subset \mathcal{A M}_{2}(f ;[a, b])$ converging uniformly to $F$ on $[a, b]$. Then each $M_{n}$ is $\underline{A C}$ on $E_{i}$. By Lemma 7, $F \in \underline{A C}$ on $E_{i}$. Similarly it follows that $-F \in \underline{A C}$ on $E_{i}$. Hence $F \in A C$ on $E_{i}$. Therefore $F \in[A C G]$ on $[a, b]$.

\section{Relations between the $\mathcal{L D G}$ and the $\mathcal{A P}_{\mathrm{j}}$ Integral}

In this section we suppose that $\mathcal{C} \subseteq \mathcal{L} \subseteq \mathcal{A} \subseteq u \mathcal{L} \subset u C M$ and that $u \mathcal{L}$ is closed under uniform convergence.

Theorem 7. Let $f:[a, b] \rightarrow \overline{\mathbb{R}}$.

(i) If $f$ is $\mathcal{L} D G$ integrable on $[a, b]$, then $f$ is $\mathcal{A P}_{j}$ integrable on $[a, b], j=$ $0,1,2$, and the integrals are equal.

(ii) If $\mathcal{A}$ is a class of the first type, then the $\mathcal{L} D G$ integral is equivalent to the $\mathcal{A P}_{j}$ integral, $j=0,1,2$.

Proof. (i) Suppose that $f$ is $\mathcal{L} D G$ integrable on $[a, b]$. Then there exists a function $F \in \mathcal{L}[a, b]$ such that $F(a)=0, F \in[A C G]$ on $[a, b]$ and $F_{a p}^{\prime}=f$ a.e. on $[a, b]$. By Lemma 5 , for $\epsilon>0$ there exists $H:[a, b] \rightarrow \mathbb{R}$ such that $H(a)=0, H(b)<\epsilon / 2, H$ is increasing and $A C$ on $[a, b]$, and $M:=F+H \epsilon$ $[(A C \cap \underline{L}) G]$ on $[a, b]$. Since $\mathcal{C} \subseteq \mathcal{L}$, it follows that $M \in \mathcal{L}[a, b] \subset \mathcal{A}[a, b]$. Thus $M \in \mathcal{A} \overline{\mathcal{M}}_{0}(f ;[a, b])$. We obtain that $M(b)<F(b)+\epsilon / 2 ;$ so

$$
\mathcal{A} \bar{I}_{0}(f ;[a, b]) \leq F(b) .
$$

Similarly, it follows that

$$
\mathcal{A} \underline{I}_{0}(f ;[a, b]) \geq F(b)
$$

Since we always have

$$
\mathcal{A} \underline{I}_{0}(f ;[a, b]) \leq \mathcal{A} \bar{I}_{0}(f ;[a, b]),
$$

we obtain that $\mathcal{A P}_{0} \int_{a}^{b} f(t) d t=F(b)$. By Corollary 3 , (i), $f$ is also $\mathcal{A} \mathcal{P}_{1}$ and $\mathcal{A P}_{2}$ integrable and the integrals are equal.

(ii) Suppose that $f$ is $\mathcal{A P}_{2}$ integrable on $[a, b]$. Let

$$
F(x):=\mathcal{A P}_{2} \int_{a}^{x} f(t) d t .
$$


By Theorem 5, $F \in \mathcal{L}[a, b]$ and $F_{a p}^{\prime}=f$ a.e. on $[a, b]$. By Theorem $6, F \in$ $[A C G]$ on $[a, b]$. Therefore $F$ is an $\mathcal{L} D G$ indefinite integral of $f$ on $[a, b]$ and

$$
\mathcal{A P}_{2} \int_{a}^{b} f(t) d t=F(b)=\mathcal{L} D G \int_{a}^{b} f(t) d t .
$$

Now see (i) and Corollary 3, (i).

Corollary 4 (A Hake-Alexandroff-Looman Type Theorem).

If $\mathcal{A}$ is a class of the first type, then the two integrals of C. M. Lee, i.e., the $\mathcal{L} D G$ integral and the $\mathcal{L} P G$ integral, are equivalent.

Proof. See Theorem 7, (ii) and Remark 6, (iv).

Corollary 5 (Special Cases).

(i) For $\mathcal{A}=u \mathcal{L}=\mathcal{C}$ the following integrals are equivalent: the $\mathcal{A P}_{0}, \mathcal{A P}_{1}$, $\mathcal{A P}_{2}$ integrals and the wide Denjoy integral.

(ii) For $\mathcal{A}=u \mathcal{L}=\mathcal{C}_{a p}$ the following integrals are equivalent: the $\mathcal{A P}_{0}, \mathcal{A P}_{1}$, $\mathcal{A P}_{2}$ integrals and the $\beta$-Ridder integral (see also Remark 6).

\section{Relations between the $\mathcal{L S}$ and the $\mathcal{A P}_{\mathrm{j}}$ Integrals}

In this section we suppose that $\mathcal{C} \subseteq \mathcal{L} \subseteq \mathcal{A} \subseteq u \mathcal{L} \subset$ lower internal and that $u \mathcal{L}$ is closed under uniform convergence.

Theorem 8 (A Hake-Alexandroff-Looman Type Theorem).

The $\mathcal{L} S$ integral is equivalent to the $\mathcal{A P}_{3}$ integral. Moreover, if the class $\mathcal{A}$ is of the second type, then the $\mathcal{L} S$ integral is equivalent to the $\mathcal{A} \mathcal{P}_{1}$ integral, and also to the $\mathcal{A P}_{2}$ integral.

Proof. Let $f:[a, b] \rightarrow \mathbb{R}$.

(I) Suppose that $f$ is $\mathcal{L} S$ integrable on $[a, b]$. Then there exists a function $F \in \mathcal{L}[a, b]$ such that $F(a)=0, F \in[V B G] \cap(N)$ on $[a, b]$ and $F_{a p}^{\prime}=f$ a.e. on $[a, b]$. It follows that $F \in \mathcal{A} \overline{\mathcal{M}}_{3}(f ;[a, b]) \times \mathcal{A M}_{3}(f ;[a, b])$. Hence $f$ is $\mathcal{A P}_{3}$ integrable on $[a, b]$ and

$$
F(b)=\mathcal{L} S \int_{a}^{b} f(t) d t=\mathcal{A} \mathcal{P}_{3} \int_{a}^{b} f(t) d t .
$$

(II) Suppose that $f$ is $\mathcal{A P}_{3}$ integrable on $[a, b]$. Let $F(x)=\mathcal{A P}_{3} \int_{a}^{x} f(t) d t$. By Theorem 5, it follows that $F_{a p}^{\prime}(x)=f(x)$ a.e. on $[a, b]$ and $F \in \mathcal{L}[a, b]$. Let $M, M_{n} \in \mathcal{A} \overline{\mathcal{M}}_{3}(f ;[a, b])$ and $m, m_{n} \in \mathcal{A M}_{3}(f ;[a, b])$ such that $M_{n} \rightarrow$ 
$F\left[\right.$ unif] and $m_{n} \rightarrow F[$ unif] on $[a, b]$. For $M$ and $m$ there exists a sequence $\left\{P_{i}\right\}_{i}$ of closed sets such that $[a, b]=\cup_{i=1}^{\infty} P_{i}, M \in V B$ and $m \in V B$ on each $P_{i}$. But the functions $M-F, M_{n}-F, F-m, F-m_{n}$ are increasing on $[a, b]$ (see Theorem 5, (i)). Then $F \in V B$ on $P_{i}$. Hence $M_{n}$ and $m_{n}$ are $V B$ on each $P_{i}$. By Lemma $8, F \in(N)$ on each $P_{i}$. Hence $F \in[V B G] \cap(N)$ on $[a, b]$. It follows that $F$ is an indefinite $\mathcal{L} S$ integral of $f$ on $[a, b]$ and

$$
F(b)=\mathcal{A P}_{3} \int_{a}^{b} f(t) d t=\mathcal{L} S \int_{a}^{b} f(t) d t .
$$

By (I) and (II) it follows that the $\mathcal{L} S$ and $\mathcal{A} \mathcal{P}_{3}$ integrals are equivalent.

We show the second part. By Corollary 3, (i), we have that the $\mathcal{A} \mathcal{P}_{1}$ and $\mathcal{A P}_{2}$ integrals are equivalent. But the $\mathcal{A P}_{3}$ integral contains the $\mathcal{A P}_{2}$ integral, and the integrals are equal (see Corollary 3 , (ii)). We also have from above that the $\mathcal{L} S$ and the $\mathcal{A P}_{3}$ integrals are equivalent. It remains to show that the $\mathcal{A P}_{2}$ integral contains the $\mathcal{L} S$ integral. Suppose that $f$ is $\mathcal{L} S$ integrable on $[a, b]$. Then there exists a function $F \in \mathcal{L}[a, b]$ such that $F(a)=0, F \in[V B G] \cap(N)$ on $[a, b]$ and $F_{a p}^{\prime}=f$ a.e. on $[a, b]$. By Lemma 6 , for $\epsilon>0$ there exist $M, m:[a, b] \rightarrow \mathbb{R}$ such that

- $M(a)=m(a)=0$ and $M(b)-m(b)<\epsilon$;

- $M \in[\underline{A C G}]$ and $m \in[\overline{A C} G]$ on $[a, b]$;

- $M-F$ and $F-m$ are increasing on $[a, b]$.

Clearly $M=(M-F)+F \in \mathcal{A}[a, b]$, because $M-F$ is increasing on $[a, b]$, $F \in \mathcal{L}[a, b] \subset \mathcal{A}[a, b]$ and $\mathcal{A}$ is a class of the second type. Similarly we obtain that $-m=(F-m)-F \in-\mathcal{A}[a, b]$. Therefore

$$
(M, m) \in \mathcal{A} \overline{\mathcal{M}}_{2}(f ;[a, b]) \times \mathcal{A \mathcal { M }}_{2}(f ;[a, b])
$$

and $M(b)<F(b)+\epsilon$ and $m(b)>F(b)-\epsilon$. It follows that $\bar{I}_{2}(f ;[a, b]) \leq$ $F(b) \leq \underline{I}_{2}(f ;[a, b])$. Since we always have that $\underline{I}_{2}(f ;[a, b]) \leq \bar{I}_{2}(f ;[a, b])$, we obtain that $\mathcal{A P}_{2} \int_{a}^{b} f(t) d t=F(b)=\mathcal{L} S \int_{a}^{b} f(t) d t$.

\section{Corollary 6.}

(i) For $\mathcal{A}=u \mathcal{L}=\mathcal{C}_{i}$ we have that $\mathcal{L}=\mathcal{C}$ and the $\mathcal{A} \mathcal{P}_{1}, \mathcal{A P}_{2}, \mathcal{A P}_{3}$ integrals and the wide Denjoy integral are equivalent.

(ii) For $\mathcal{A}=u \mathcal{L}=\mathcal{C}_{i, a p}$ we have that $\mathcal{L}=\mathcal{C}_{a p}$, and the integrals $\mathcal{A P}_{1}, \mathcal{A P}_{2}$, $\mathcal{A P}_{3}$ and $\mathcal{L} S$ are equivalent. But the $\mathcal{L} S$ integral is a strict generalization of C. M. Lee's $\mathcal{L} D G$ integral.

(iii) C. M. Lee's $\mathcal{L} P G$ integral is a strict generalization of his $\mathcal{L} D G$ integral. 
Proof. (i) See Theorem 8.

(ii) That $\mathcal{L} S \supset \mathcal{L} D G$ follows immediately from definitions. We show that the inclusion is proper. By Remark 3 , for $\mathcal{L}=\mathcal{C}_{a p}$, the $\mathcal{L} D G$ integral is the $\beta$-Ridder integral. By Remark 4, (iv), we obtain that $\mathcal{L} D G \neq \mathcal{L} S$.

(iii) By Theorem 7, (i), it follows that $\mathcal{L} D G \subset \mathcal{A P}_{2}$. Assuming the hypotheses of (ii), we obtain that $\mathcal{L} P G=\mathcal{A} \mathcal{P}_{2}=\mathcal{L} S \neq \mathcal{L} D G$.

\section{Relations between the $\mathcal{L S G}$ and the $\mathcal{A P}_{4}$ Integrals}

In this section we suppose that $\mathcal{C} \subseteq \mathcal{L} \subseteq \mathcal{A} \subseteq u \mathcal{L} \subset$ lower internal and that $u \mathcal{L}$ is closed under uniform convergence.

Theorem 9 (A Hake-Alexandroff-Looman Type Theorem).

The $\mathcal{L} S G$ integral is equivalent to the $\mathcal{A P}_{4}$ integral.

Proof. Let $f:[a, b] \rightarrow \mathbb{R}$. (I) Suppose that $f$ is $\mathcal{L} S G$ integrable on $[a, b]$. Then there exists a function $F \in \mathcal{L}[a, b]$ such that $F(a)=0, F \in V B G \cap$ $\mathcal{B}_{1} \cap(N)$ on $[a, b]$ and $F_{a p}^{\prime}=f$ a.e. on $[a, b]$. Then $F \in \mathcal{A} \overline{\mathcal{M}}_{4}(f ;[a, b]) \times$ $\mathcal{A M}_{4}(f ;[a, b])$. Hence

$$
(\mathcal{L} S G) \int_{a}^{b} f(t) d t=F(b)=\mathcal{A P}_{4} \int_{a}^{b} f(t) d t .
$$

(II) Suppose that $f$ is $\mathcal{A P}_{4}$ integrable on $[a, b]$. Let $F(x)=\mathcal{A P}_{4} \int_{a}^{x} f(t) d t$. By Theorem 5, it follows that $F_{a p}^{\prime}(x)=f(x)$ a.e. on $[a, b]$ and $F \in \mathcal{L}[a, b]$. Let $M, M_{n} \in \mathcal{A} \overline{\mathcal{M}}_{4}(f ;[a, b])$ and $m, m_{n} \in \mathcal{A M}_{4}(f ;[a, b])$ such that $M_{n} \rightarrow$ $F$ [unif] and $m_{n} \rightarrow F$ [unif] on $[a, b]$. For $M$ and $m$ there exists a sequence $\left\{P_{i}\right\}_{i}$ such that $[a, b]=\cup_{i=1}^{\infty} P_{i}, M \in V B$ and $m \in V B$ on each $P_{i}$. We may suppose that each $P_{i}$ is a Borel set. Indeed, there exists a function $\tilde{M}_{i}:[a, b] \rightarrow \mathbb{R}$ such that $\left(\tilde{M}_{i}\right)_{\mid P_{i}}=M$ and $\tilde{M}_{i} \in V B$ on $[a, b]$ (see [19], p. 221). Let $Q_{i}=\left\{x: \tilde{M}_{i}(x)=M(x)\right\}$. Then $P_{i} \subset Q_{i}$ and $Q_{i}$ is a Borel set (because $M$ and $\tilde{M}_{i}$ are $\mathcal{B}_{1}$ on $[a, b]$ ).

But the functions $M-F, M_{n}-F, F-m, F-m_{n}$ are increasing on $[a, b]$ (see Theorem 5, (i)). Then $F \in V B$ on $P_{i}$. Hence $M_{n}$ and $m_{n}$ are $V B$ on each $P_{i}$. By Lemma 8, $F \in(N)$ on each $P_{i}$. Hence $F \in V B G \cap(N)$ on $[a, b]$. Since $M \in \mathcal{B}_{1}$ on $[a, b]$ and $M-F$ is increasing on $[a, b]$, it follows that $F \in \mathcal{B}_{1}$ on $[a, b]$. It follows that $F$ is an indefinite $\mathcal{L} S G$ integral of $f$ on $[a, b]$ and $F(b)=\mathcal{A P}_{4} \int_{a}^{b} f(t) d t=(\mathcal{A} S G) \int_{a}^{b} f(t) d t$.

\section{References}

[1] A. M. Bruckner, Differentiation of real functions, Lect. Notes in Math., vol. 659, Springer-Verlag, 1978. 
[2] V. Ene, Real functions - current topics, Lect. Notes in Math., vol. 1603, Springer-Verlag, 1995.

[3] V. Ene, On Borel measurable functions that are $V B G$ and $(N)$, Real Analysis Exchange 22 (1996-1997), no. 2, 688-695.

[4] V. Ene, Lusin's condition $(N)$ and Foran's condition $(M)$ are equivalent for Borel functions that are VBG on a Borel set, (submitted).

[5] J. Foran, A generalization of absolute continuity, Real Analysis Exchange 5 (1979-1980), 82-91.

[6] K. M. Garg, A new notion of derivative, Real Analysis Exchange 7 (19811982), 65-84.

[7] R. Gordon, Some comments on an approximately continuous Khintchine integral, Real Analysis Exchange 20 (1994-95), no. 2, 831-841.

[8] Y. Kubota, An integral of Denjoy type, Proc. Japan Acad. 40 (1964), $713-717$.

[9] Y. Kubota, An integral of Denjoy type. II, Proc. Japan Acad. 42 (1966), no. $7,737-742$.

[10] Y. Kubota, A characterization of the approximately continuous Denjoy integral, Can. J. Math. 22 (1970), 219-226.

[11] C. M. Lee, An analogue of the theorem Hake-Alexandroff-Looman, Fund. Math. C (1978), 69-74.

[12] C. M. Lee, On Baire one Darboux functions with Lusin's condition (N), Real Analysis Exchange 7 (1981), 61-64.

[13] I. P. Natanson, Theory of functions of a real variable, 2nd. rev. ed., Ungar, New York, 1961.

[14] J. Ridder, Über den Perronschen Integralbegriff und seine Beziehung zu den $R$-, L-, und D- Integralen, Math. Zeit. 34 (1931), 234-269.

[15] J. Ridder, Über approximativ stetige Denjoy-Integrale, Fund. Math. 21 (1933), 1-10.

[16] J. Ridder, Über das allgemeine Denjoysche-Integral, Fund. Math. 21 (1933), 11-19.

[17] J. Ridder, Über die gegenseitigen Beziehungen verschiedener approximativ stetigen Denjoy-Perron Integrale, Fund. Math. 22 (1934), 136-162. 
[18] J. Ridder, Über die T- und N-Bedingungen und die approximativ stetigen Denjoy-Perron Integrale, Fund. Math. 22 (1934), 163-179.

[19] S. Saks, Theory of the integral, 2nd. rev. ed., vol. PWN, Monografie Matematyczne, Warsaw, 1937.

[20] D. N. Sarkhel, A wide Perron integral, Bull. Austral. Math. Soc. 34 (1986), 233-251.

[21] D. N. Sarkhel, A wide constructive integral, Math. Japonica 32 (1987), 295-309.

[22] D. N. Sarkhel and A. K. De, The proximally continuous integrals, J. Austral. Math. Soc. (Series A) 31 (1981), 26-45.

[23] D. N. Sarkhel and B. Kar, (PVB) functions and integration, J. Austral. Math. Soc. (Series A) 36 (1984), 335-353.

[24] D. E. Varberg, On absolutely continues functions, Amer. Math. Monthly 72 (1965), 831-841. 